Research Article

\title{
Complex Dynamics and Hard Limiter Control of a Fractional-Order Buck-Boost System
}

\author{
Bo Yan $\left(\mathbb{D},{ }^{1}\right.$ Shaojie Wang $\mathbb{D},{ }^{1}$ and Shaobo He $\mathbb{D}^{2}$ \\ ${ }^{1}$ College of Electrical and Information Engineering, Shaoyang University, Shaoyang 422000, China \\ ${ }^{2}$ School of Physics and Electronics, Central South University, Changsha 410083, China \\ Correspondence should be addressed to Shaobo He; heshaobo_123@163.com
}

Received 4 February 2021; Revised 27 February 2021; Accepted 4 March 2021; Published 19 March 2021

Academic Editor: Rongwei Guo

Copyright (C) 2021 Bo Yan et al. This is an open access article distributed under the Creative Commons Attribution License, which permits unrestricted use, distribution, and reproduction in any medium, provided the original work is properly cited.

Chaos and control analysis for the fractional-order nonlinear circuits is a recent hot topic. In this study, a fractional-order model is deduced from a Buck-Boost converter, and its discrete solution is obtained based on the Adomian decomposition method (ADM). Chaotic dynamic characteristics of the fractional-order system are investigated by the bifurcation diagram, $0-1$ test, spectral entropy (SE) algorithm, and NIST test. Meanwhile, the control of the fractional-order Buck-Boost model is discussed through two different ways, namely, the intensity feedback and the hard limiter control. Specifically, the hard limiter control can be realized using a current limiter in the circuit, where the current limiter device is applied to control the branch current. The results show that the proposed fractional-order system has complex dynamic behaviors and potential application values in the engineering field.

\section{Introduction}

The Buck-Boost converters are strongly nonlinear circuits [1-4] which can produce subharmonic, bifurcation, and chaos under certain conditions. At present, many researchers have investigated the chaos of the Buck-Boost converter systems [5-11]. For example, Wang et al. [10] investigated the mechanisms that lead to chaotic behaviors in the Buck-Boost power converter, and the existence of chaos is verified successfully by the Smale horseshoe, while Demirbaş et al. [11] investigated the bifurcation of the system with the material parameters and found a number of periodic windows. Obviously, chaos will lead to the instability of the converter via increasing the oscillation and producing the excessive irregular electromagnetic noise, which directly affects the operation quality and reliability of the converter. In fact, the Buck-Boost is a switched model, and control of the nonlinear switched systems is a challenging topic [12]. As a result, there are also many other reports about the chaos control of the Buck-Boost converters [13-15]. For instance, Sriramalakshmi et al. [15] analyzed the nonlinear phenomena and control of the current mode- controlled Buck-Boost converter. Meanwhile, complexity of nonlinear dynamical systems has aroused much interest of researchers [16, 17]. Therefore, an in-depth study of the complexity with the circuit parameters is still significant for the design and application of the Buck-Boost converter systems.

The fractional calculus was proposed at about the same time as the regular calculus. At present, since it can better describe those processes with time and the space memory effect, it has been accepted as a novel tool for building the mathematical models. Nowadays, the fractional calculus has extensive applications in the field of physics, engineering, and biology economics [18-20]. Generally, the nonlinear systems have more complex dynamical behaviors after the fractional calculus is introduced [21-24]. In fact, dynamics analysis of different kinds of fractional-order Buck-Boost systems has aroused concern from scholars [25-28]. However, these studies mainly focused on the modeling of fractional-order Buck-Boost systems. Currently, dynamics in different kinds of nonlinear systems have been investigated, such as memristor-based systems $[29,30]$ and chaotic systems [31, 32]. It is indicated that those systems have 
abundant dynamic characteristics including the multistability and can be used for the image encryption applications. However, dynamics of the fractional-order BuckBoost systems still need further study. Meanwhile, how to build an effective model is necessary for the applications of the fractional-order Buck-Boost system. In fact, He et al. [33] shows that, compared with other solution algorithms, the Adomian decomposition method (ADM) is more accurate, and the deduced solution can be used in the engineering applications. Thus, it can be employed to solve fractionalorder Buck-Boost systems for further analysis and applications.

In 2008, Wu and Zhang [34] proposed an improved exponential delayed feedback controller to a Buck-Boost converter in the current mode. This exponential delayed feedback controller provides a new strategy for chaos control and for the switching law. As a matter of fact, control of chaos is important for the application of nonlinear systems [35]. On the other hand, although the chaotic systems can be applied in secret communications and information encryption [36,37], there are few reports on applications of the Buck-Boost system in information security field. Motivated by the above discussions, we will explore the dynamics and control the potential application values of the fractionalorder Buck-Boost system based on $\mathrm{Wu}$ and Zhang's model [34] by employing the ADM.

The rest of the study is organized as follows. In Section 2, the fractional-order Buck-Boost model which is a switching system is solved by employing the ADM. In Section 3, complexity of the fractional-order Buck-Boost system is investigated, and the existence of chaos is verified by the 0-1 test. A chaotic pseudorandom sequence generator is designed. In Section 4, control of chaos in the fractionalorder Buck-Boost system is studied. Two different approaches are introduced. Finally, conclusion is made in Section 5 .

\section{The Fractional-Order Buck-Boost Model}

2.1. Building the Fractional-Order Buck-Boost System. Wu and Zhang [34] designed a Buck-Boost converter in the current mode and found chaos in the proposed model. The circuit of the modified Buck-Boost converter is shown in Figure 1, and the description of the parameters is given in Table 1. In this enhanced Buck-Boost converter, there is an adjustment coefficient $k$ which can be used to control the stability of the converter.

Let the Buck-Boost converter work in the continuous conductive mode, and the inductance current $x_{1}\left(i_{L}\right)$ and the capacitance voltage $x_{2} \quad\left(u_{c}\right)$ be the system variables. According to on and off states of the power switch in the converter and based on Kirchhoff's law, the Buck-Boost converter system can be expressed as [34]

$$
\left\{\begin{array} { l } 
{ \dot { x } _ { 1 } = \frac { 1 } { L } U _ { \text { in } } , } \\
{ \dot { x } _ { 2 } = - \frac { 1 } { R C } x _ { 2 } , }
\end{array} \left\{\begin{array}{l}
\dot{x}_{1}=-\frac{1}{L} x_{2}, \\
\dot{x}_{2}=\frac{1}{C} x_{1}-\frac{1}{R C} x_{2} .
\end{array}\right.\right.
$$
38]

Now, the Caputo fractional calculus, which is given by

$$
D_{t_{0}}^{q} f(t)=\frac{1}{\Gamma(1-q)} \int_{t_{0}}^{t}(t-\tau)^{-q} f^{\prime}(\tau) \mathrm{d} \tau,
$$

is introduced to the system, where $D_{t_{0}}^{q}$ is the Caputo derivative operator of order $q(0<q \leq 1)$, $t_{0}$ is the initial time, and $\Gamma(\cdot)$ is the Gamma function. Thus, the fractional-order Buck-Boost system is defined as

$$
\begin{aligned}
& \left\{\begin{array}{l}
D_{t_{0}}^{q} x_{1}=\frac{1}{L} U_{\text {in }}, \\
D_{t_{0}}^{q} x_{2}=-\frac{1}{R C} x_{2},
\end{array}\right. \\
& \left\{\begin{array}{l}
D_{t_{0}}^{q} x_{1}=-\frac{1}{L} x_{2}, \\
D_{t_{0}}^{q} x_{2}=\frac{1}{C} x_{1}-\frac{1}{R C} x_{2} .
\end{array}\right.
\end{aligned}
$$

Although there are some research studies regarding the fractional-order Buck-Boost system, it is necessary to give more interpretations of the fractional-order Buck-Boost system [25-28]. Usually, we hold the opinion that the fractional-order calculus provides a more effective way for modeling of real systems, and the analysis results can reflect the dynamics of real systems better. Here, we hope that the fractional-order system could provide more information about the Buck-Boost circuit.

2.2. The Adomian Decomposition Method. As shown above, the systems have only linear items and a constant. To solve this system, the ADM [39-41] is employed, and the description of this algorithm is illustrated as follows.

For a given fractional-order linear system,

$$
\left\{\begin{array}{l}
D_{t_{0}}^{q} \mathbf{x}(t)=\Upsilon \mathbf{x}+\mathbf{g} \\
\dot{\mathbf{x}}\left(t_{0}^{+}\right)=\mathbf{x}_{0},
\end{array},\right.
$$

where $D_{t_{0}}^{q}$ is the Caputo definition, $\Upsilon \mathbf{x}$ is the linear terms in the system, and $q \in(0,1]$. Its solution is given by [39]

$$
\mathbf{x}=\sum_{i=1}^{\infty} \mathbf{x}^{i}=J_{t_{0}}^{q} \Upsilon \sum_{i=0}^{\infty} \mathbf{x}^{i}+J_{t_{0}}^{q} \mathbf{g}+\mathbf{x}_{0} .
$$

Meanwhile, those decomposition items $x^{i} \quad(i=0,1$, $\ldots, \infty)$ are calculated as $[40,41]$

$$
\left\{\begin{array}{l}
\mathbf{x}^{0}=J_{t_{0}}^{q} \mathbf{g}+\mathbf{x}_{0}, \\
\mathbf{x}^{i}=J_{t_{0}}^{q} \Upsilon \mathbf{x}^{i-1}, \quad i=1,2, \ldots, \infty
\end{array}\right.
$$

When an infinite number of items is used, the solution is the exact solution. In this study, 11 decomposition items, namely, $x^{i}(i=0,1, \ldots, 10)$, are used. 
2.3. Solution of the Fractional-Order Model. Let us focus on the $n^{\text {th }}$ sampling period; thus, $t \in\left(t_{n}, t_{n+1}\right]$. The initial value of this interval is $x_{1}\left(t_{n}\right)$ and $x_{2}\left(t_{n}\right)$.

Case 1. By employing the above ADM, the solution of equation (3) is given by

$$
\left\{\begin{array}{l}
x_{1}(t)=x_{1}\left(t_{n}\right)+\frac{U_{\mathrm{in}}}{L} \frac{\left(t-t_{n}\right)^{q}}{\Gamma(q+1)} \\
x_{2}(t)=x_{2}\left(t_{n}\right) \sum_{i=0}^{10} \kappa_{2}^{i} \frac{\left(t-t_{n}\right)^{\mathrm{iq}}}{\Gamma(\mathrm{iq}+1)}
\end{array}\right.
$$

where

$$
\left\{x_{1}(t)=x_{1}\left(t_{n}\right)+\sum_{i=1}^{10} c_{1}^{i} \frac{\left(t-t_{n}\right)^{\mathrm{iq}}}{\Gamma(\mathrm{iq}+1)}, x_{2}(t)=x_{2}\left(t_{n}\right)+\sum_{i=1}^{10} c_{2}^{i} \frac{\left(t-t_{n}\right)^{\mathrm{iq}}}{\Gamma(\mathrm{iq}+1)}\right.
$$

where

$$
\begin{aligned}
& \left\{\begin{array}{l}
c_{1}^{i}=-\frac{1}{L} c_{2}^{i-1}, \\
c_{2}^{i}=\frac{1}{C} c_{1}^{i-1}-\frac{1}{R C} c_{2}^{i-1},
\end{array}\right. \\
& c_{1}^{0}=x_{1}\left(t_{n}\right), \\
& c_{2}^{0}=x_{2}\left(t_{n}\right) .
\end{aligned}
$$

So the following discrete solution is obtained:

$$
\mathbf{x}\left(t_{n+1}\right)=f_{2}\left(\mathbf{x}\left(t_{n}\right), h, q\right) .
$$

Based on $x_{1}\left(t_{n}\right)$ and $x_{2}\left(t_{n}\right)$, the reference current for the inductance $I_{\text {ref }}\left(t_{n}\right)$, the boundary value of the inductance current $I_{b}\left(t_{n}\right)$, and the duty cycle $d\left(t_{n}\right)$ are calculated by [34]

$$
\left\{\begin{aligned}
I_{\text {ref }}\left(t_{n}\right) & =I_{\text {ref }}-e^{k\left(u_{n}-u_{n-1}\right)}+1, \\
I_{b}\left(t_{n}\right) & =I_{\text {ref }}\left(t_{n}\right)-\frac{U_{\text {in }} T}{L} \\
d\left(t_{n}\right) & =L \frac{I_{\text {ref }}\left(t_{n}\right)-x_{1}\left(t_{n}\right)}{U_{\text {in }} T} .
\end{aligned}\right.
$$

When $x_{1}\left(t_{n}\right)<I_{b}\left(t_{n}\right)$, the power switch $S$ is on during the whole sampling period, and the system is defined as Case 1 . However, if $x_{1}\left(t_{n}\right) \geq I_{b}\left(t_{n}\right)$, the power switch $S$ is

$$
\begin{aligned}
& \kappa_{2}^{i}=-\frac{1}{R C} \kappa_{2}^{i-1}, \\
& \kappa_{2}^{0}=1 .
\end{aligned}
$$

When $t=t_{n+1}$, we can obtain the following discrete solution of the system, which is defined as

$$
x\left(t_{n+1}\right)=f_{1}\left(x\left(t_{n}\right), h, q\right) .
$$

Case 2. By employing the above ADM, the solution of equation (4) is denoted as 


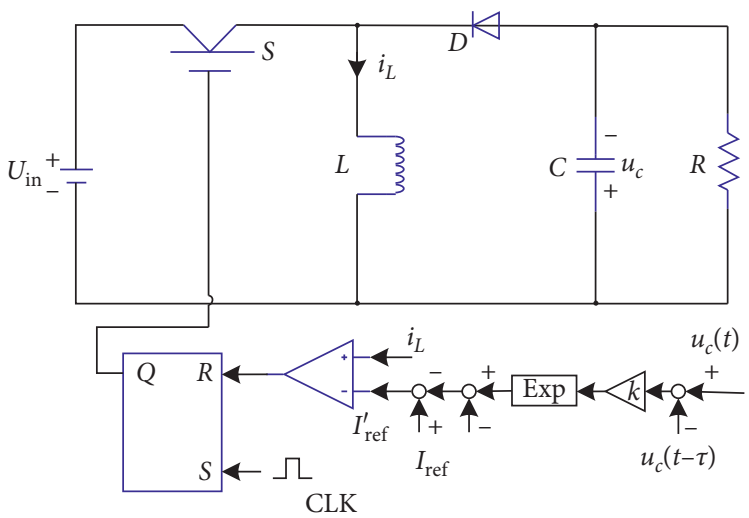

Figure 1: Circuit of the Buck-Boost converter.

TABLE 1: Description of the parameters in the Buck-Boost converter.

\begin{tabular}{lc}
\hline Symbol & Explanation \\
\hline$U_{\text {in }}$ & The input voltage of the converter \\
$S$ & The power switch \\
$L$ & The diode \\
$C$ & The inductance \\
$R$ & The capacitance \\
$i_{L}$ & The load resistance \\
$u_{c}$ & The inductive current \\
$I_{\text {ref }}$ & The capacitor voltage \\
$x_{1}$ and $x_{2}$ & The inductance reference current \\
$k$ & The inductive current $\left(i_{L}\right)$ and the capacitor voltage $\left(u_{c}\right)$ \\
\hline
\end{tabular}

inductance reference current $L$, the intensity of feedback $k$, and the derivative order $q$. Figure 3 shows the bifurcation diagrams with the inductance reference current $I_{\text {ref }}$ under a different derivative order $q$. In Figure $3, I_{\text {ref }}$ varies from 0 to 4 with step size of 0.008 and $k=0$. As shown in Figure 3, dynamics of the system with the variation of $I_{\text {ref }}$ are different with a different derivative order $q$. Meanwhile, bifurcations versus different parameters are analyzed. Fix $k=0, q=0.9$, and $I_{\text {ref }}=3 \mathrm{~A}$, and vary $L$ from $2 \mathrm{mH}$ to $5 \mathrm{mH}$ with step size of 0.006. The analysis result is shown in Figure 4(a). Fix $q=0.9, I_{\text {ref }}=3 \mathrm{~A}$, and $L=0.3 \mathrm{mH}$, and vary parameter $k$ from 0 to 5 with step size of 0.01 . The bifurcation diagram with $k$ is shown in Figure $4(\mathrm{~b})$. Let $U_{\text {in }}=8 \mathrm{~V}, L=0.3 \mathrm{mH}$, $R=10 \Omega, C=40 \mu \mathrm{F}, k=0$, and $T=50 \mu \mathrm{s}$, and vary the derivative order $q$ from 0.85 to 1 , where the step size is $3 \times 10^{-4}$. As shown in Figure 4, the system has rich dynamics with those parameters. The systems can be chaotic and periodic with different parameters. For instance, when the intensity of feedback $k$ is larger than 2 , the system is periodic. It means that the system is nonchaotic, and $k$ can be treated as a control parameter of the circuit.

3.2. $0-1$ Test. The proposed system is a typical switched system, and Lyapunov exponents of the system cannot be calculated directly. In this study, the $0-1$ test is employed to verify the existence of chaos.
If a set of one-dimensional observable data obtained from the iterative is represented by a set of multiscale discrete time series $\{x(n): n=1,2,3, \ldots\}$, then the following two real valued sequences can be defined as $[42,43]$

$$
\left\{\begin{array}{l}
p(n)=\sum_{j=1}^{n} x(j) \cos (\theta(j)) \\
s(n)=\sum_{j=1}^{n} x(j) \sin (\theta(j))
\end{array}\right.
$$

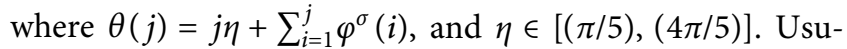
ally, the state of the system can be identified by plotting the trajectories in the $(p, s)$ plane. Figure 5 shows the $(p, s)$ plots of the system with a different derivative order $q$, where the parameters are set as same as Figure 1, respectively. It shows that the system is chaotic when $q=1,0.95$, and 0.9 , while the system is periodic when $q=0.85$. Thus, the existence of chaos is verified.

However, $(p, s)$ plots can only be used to illustrate the state of the system under given parameters but cannot be used to show dynamics with the variation of parameters. Thus, it is necessary to deduce an index from the $(p, s)$ plots. Fortunately, Gottwald and Melbourne [43] proposed a test which can be used to distill a binary quantity $(K)$ from the power spectrum. Namely, if $K=0$, the time series has regular dynamics, but if $K=1$, the time series is chaotic. 


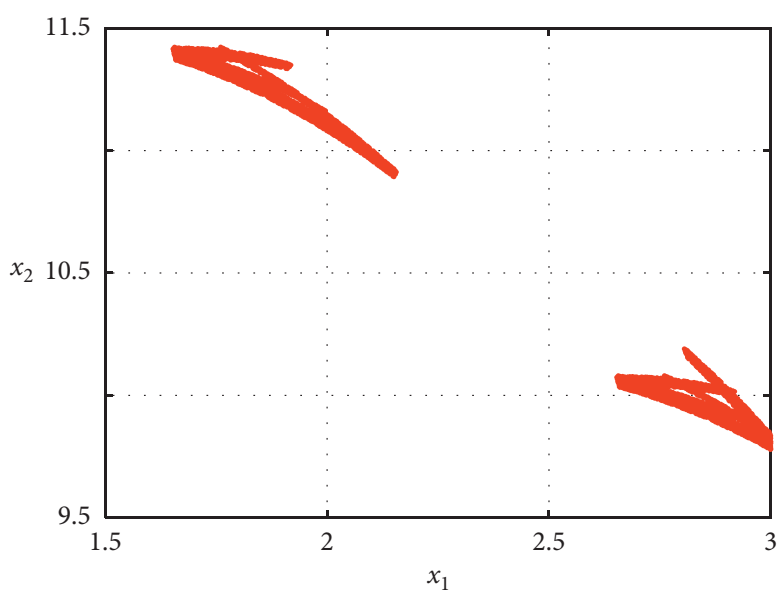

(a)

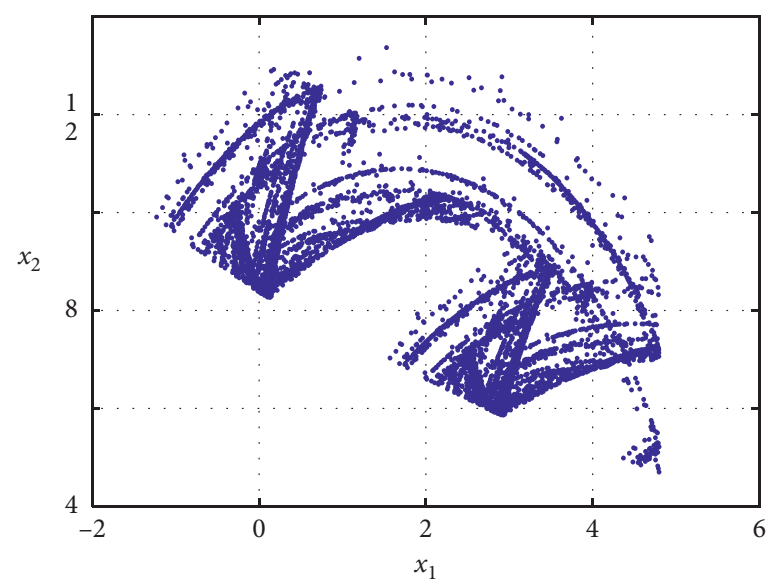

(c)

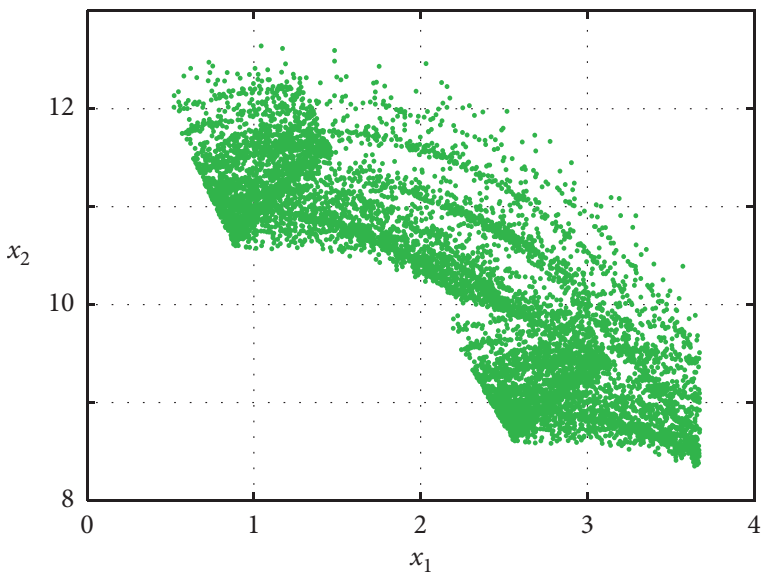

(b)

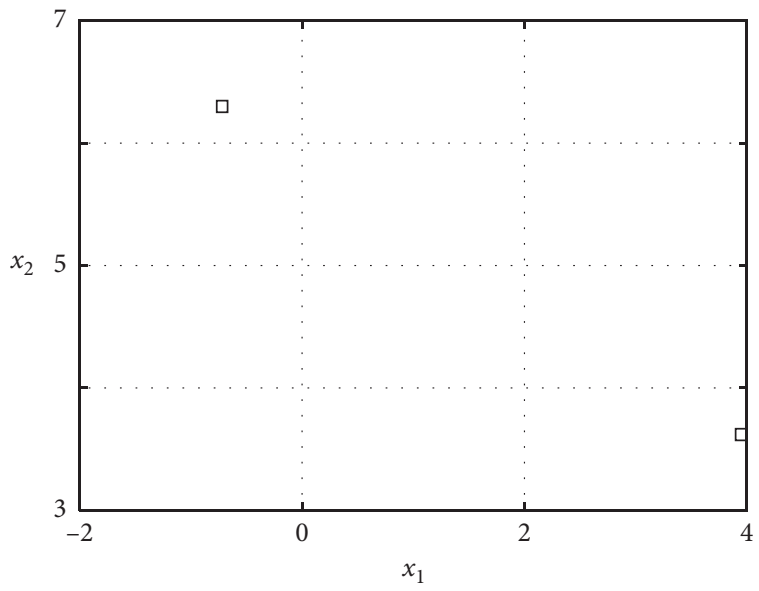

(d)

Figure 2: Phase diagrams of the fractional-order model. (a) $q=1$, (b) $q=0.95$, (c) $q=0.9$, and (d) $q=0.85$.

There are two steps for this quantity which are expanded as follows.

First, the mean square displacement is defined as $[42,43]$

$$
M(n)=\lim _{N \longrightarrow \infty} \frac{1}{N} \sum_{j=1}^{N}\left[(p(j+n)-p(j))^{2}+(s(j+n)-s(j))^{2}\right],
$$

where $n \ll N$. In real applications, $n \leq(N / 10)$ yields good results.

Second, the asymptotic growth rate $K$ is defined as $[42,43]$

$$
K=\lim _{n \rightarrow \infty} \frac{\log (M(n))}{\log (n)} .
$$

Plots of $K$ versus different parameters for the fractionalorder Buck-Boost system are shown in Figure 6. Figures 6(a)-6(d) show the dynamics corresponding to the bifurcation diagrams as shown in Figure 3. And Figures $6(\mathrm{e})-6(\mathrm{~g})$ are the corresponding analysis results of Figure 4 with the variation of parameters $L, k$, and $q$, respectively. It is shown in Figure 6 that chaos in the system is verified since $K$ reaches to 1 , and the $K$ curves agree well with the bifurcation diagrams.
Moreover, $K$-value based contour plots of the fractionalorder Buck-Boost system in different parameter planes is shown in Figure 7, where those parameter planes are divided as the $100 \times 100$ grid. The yellow color means that the system is chaotic when it takes parameters in those regions, while the rest parts mean that the system is nonchaotic. Meanwhile, it also shows that the system has wide regions for chaos in those parameter planes.

3.3. Complexity Analysis. Bifurcation diagrams, phase diagrams, $(p, s)$ plot, and $K$ curves are mainly used to analyze dynamics of the system but cannot show how the complexity of the system changes with the parameters. The spectral entropy (SE) [33] algorithm is employed to analyze complexity of the fractional-order Buck-Boost system, and details of this algorithm are presented as follows.

Given a time sequence of length $N\{x(n), n=0,1,2, \ldots$, $N-1\}$, let $x(n)=x(n)-\bar{x}$, where $\bar{x}$ is the mean value of time series. Its corresponding DFT is defined by

$$
X(k)=\sum_{n=0}^{N-1} x(n) e^{(-j 2 \pi n k / N)},
$$




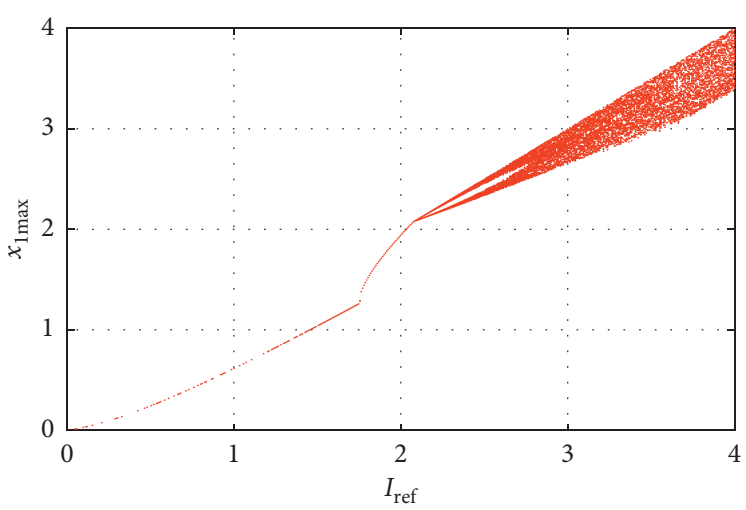

(a)

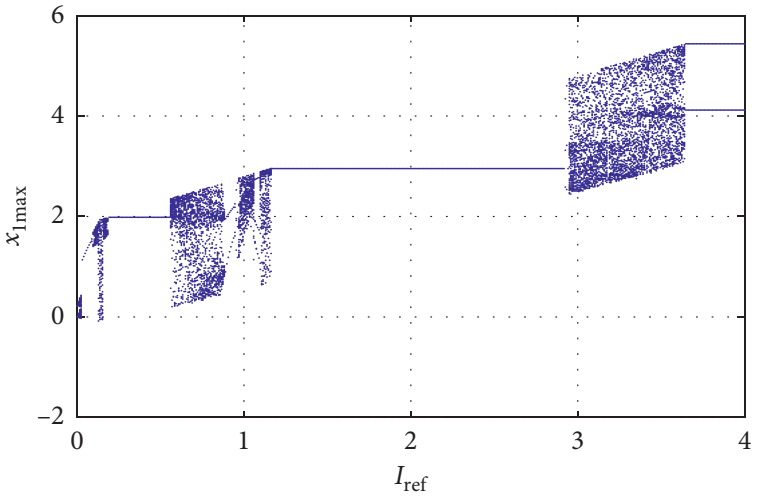

(c)

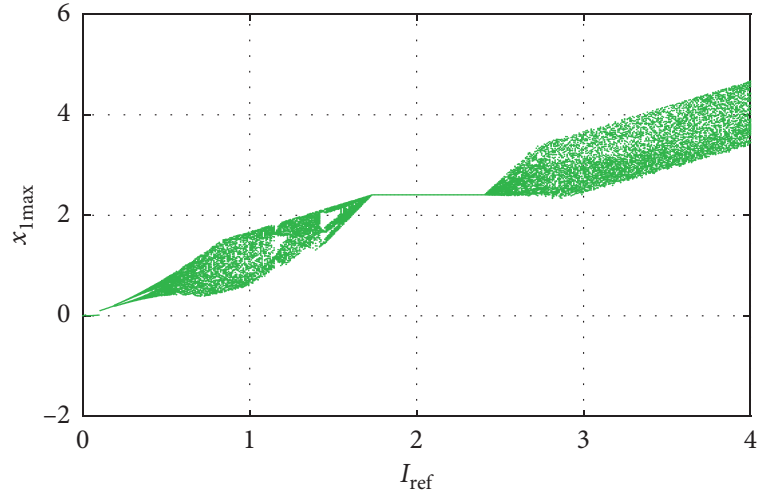

(b)

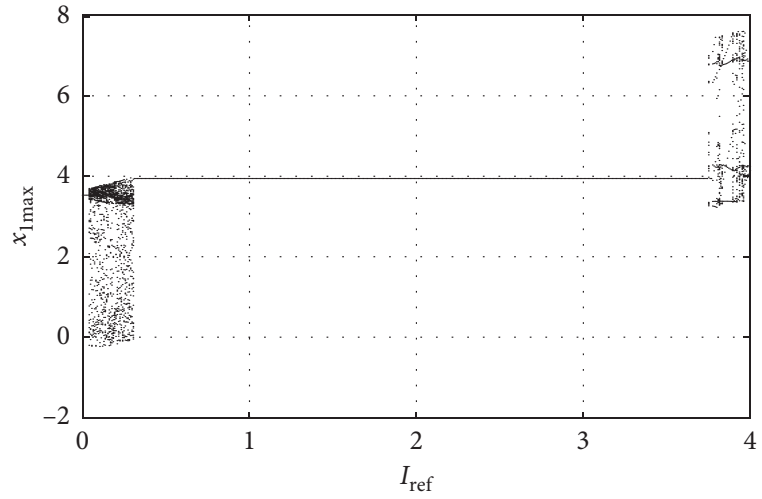

(d)

FIGURE 3: Bifurcation diagrams with the inductance reference current $I_{\text {ref }}$ under different derivative orders $q$. (a) $q=1$, (b) $q=0.95$, (c) $q=0.9$, and (d) $q=0.85$.

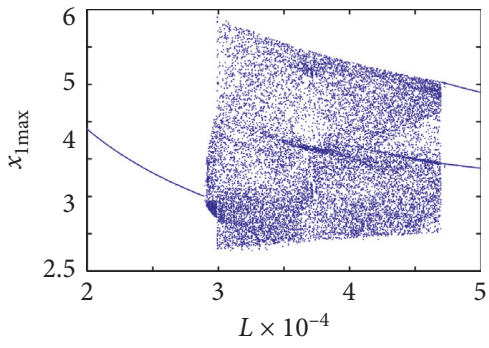

(a)

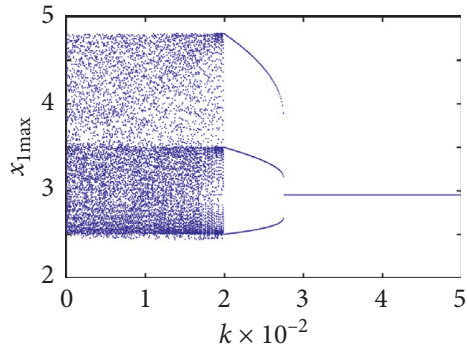

(b)

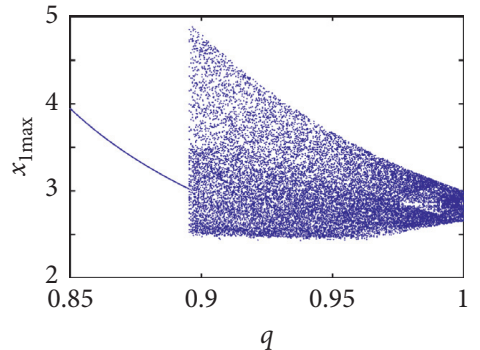

(c)

FIGURE 4: Bifurcation diagrams with the variation of different parameters. (a) $L$ varying, (b) $k$ varying, and (c) $q$ varying.

where $k=0,1, \ldots, N-1$, and $j$ is the imaginary unit. If the power of a discrete power spectrum with the $k_{\mathrm{th}}$ frequency is $|X(k)|^{2}$, then the "probability" of this frequency is defined as

$$
P_{k}=\frac{|X(k)|^{2}}{\sum_{k=0}^{N /(2-1)}|X(k)|^{2}} .
$$

When the DFT is employed, the summation runs from $k=0$ to $k=(N /(2-1))$. The normalization entropy is denoted by [33]

$$
S E\left(x^{N}\right)=\frac{1}{\ln (N / 2)} \sum_{k=0}^{N /(2-1)} P_{k} \ln \left(P_{k}\right),
$$




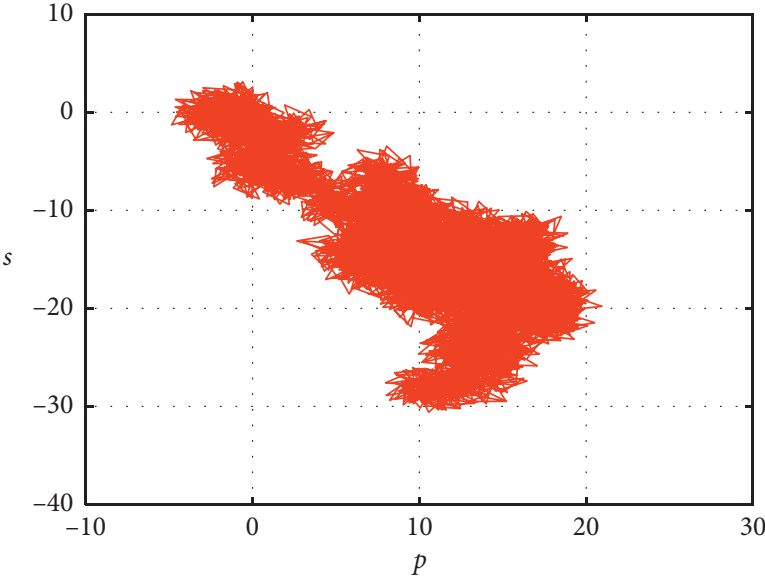

(a)

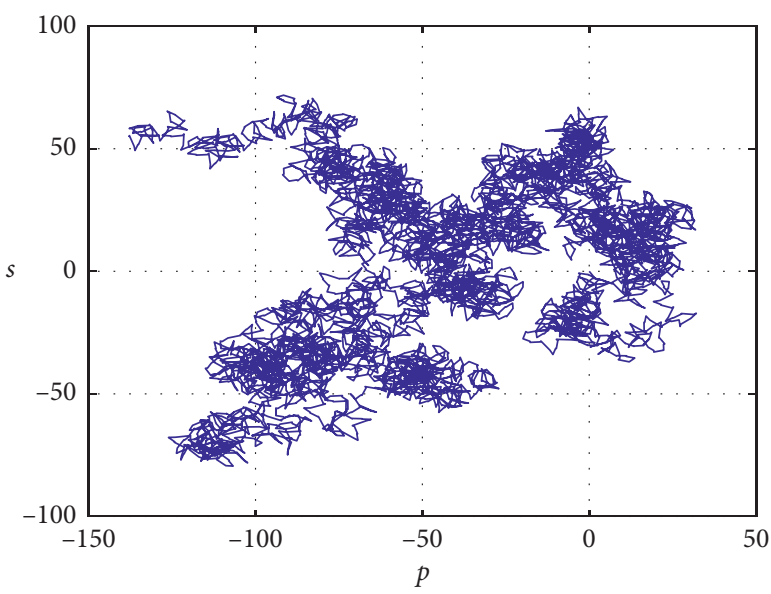

(c)

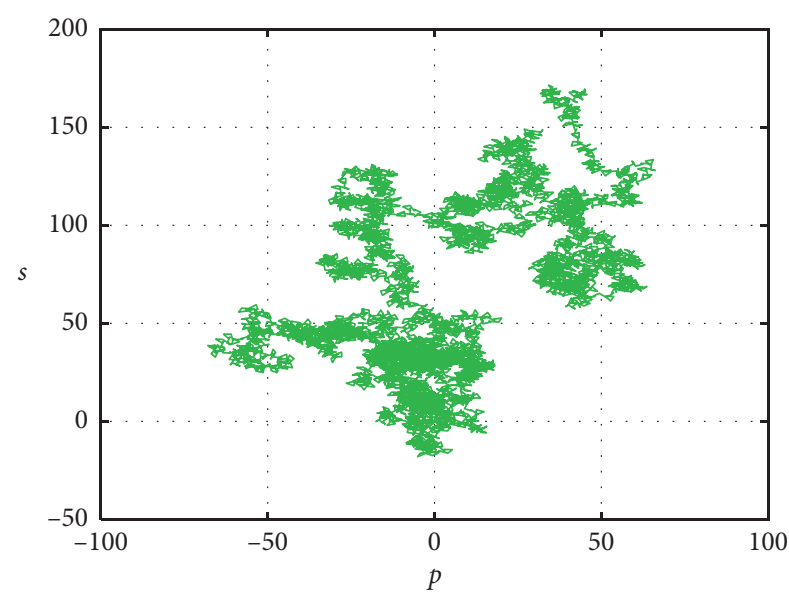

(b)

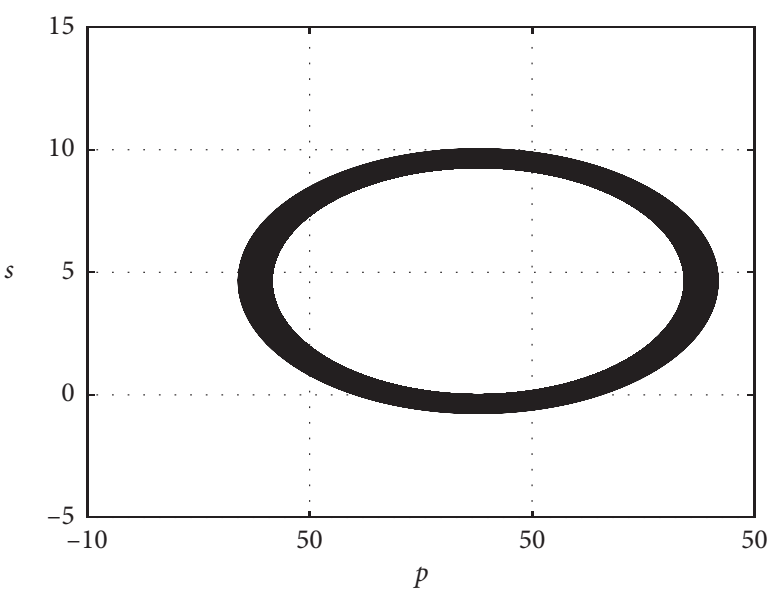

(d)

Figure 5: $(p, s)$ plots of the fractional-order model. (a) $q=1$, (b) $q=0.95$, (c) $q=0.9$, and (d) $q=0.85$.

where $\ln (N / 2)$ is the entropy of a completely random signal. Obviously, the more balanced the probability distribution is, the higher complexity (the larger entropy) the time series is. The larger measuring value means higher complexity and vice versa.

Using the same parameters, SE complexity of the fractional-order Buck-Boost system with different parameters is analyzed, and the results are shown in Figures 8 and 9. First, the SE analysis results have a high positive correlation with the corresponding bifurcation diagrams and 0-1 test results. As shown Figures 8 and 9, higher complexity is observed when the system is chaotic, but the nonchaotic states have lower complexity analysis results. Second, compared with the other methods in this study, SE complexity shows the variation tendency when the parameters vary.

3.4. Chaotic Pseudorandom Sequence Generator. Chaotic behaviors such as randomness, sensitive dependence on initial conditions, and ergodicity are important issues for the real applications. At present, there are many researchers focusing on the applications of chaos, such as chaos-based application of a novel no-equilibrium chaotic system with coexisting attractors [44], chaotic artificial neural networks for model memory in the brain [45], and a fractional-order chaotic system with an infinite number of equilibrium points located on a line and on a hyperbola [46]. Currently, designing chaos-based pseudorandom sequence generators has aroused much interest of researchers [33, 47-49]. Since the fractional-order Buck-Boost system has high complexity, it is a good model for designing the chaotic pseudorandom sequence generator.

In this section, by modifying our previously designed pseudorandom sequence generator [33], a pseudorandom bit generator is designed based on the fractional-order BuckBoost system. The specific steps are given as follows.

Step 1. Set $U_{\text {in }}=8 \mathrm{~V}, R=10 \Omega, L=0.3 \mathrm{mH}, C=40 \mu \mathrm{F}$, $T=50 \mu \mathrm{s}, I_{\text {ref }}=4 \mathrm{~A}, k=0$, and $q=0.95$. The initial value $\mathbf{x}_{0}=[1,2], M=0.125 \times 10^{8}+100$, and iterate the system 1000 times; then $n=1000$, and thus, $\mathbf{x}_{0}=\left[x_{1}(n), x_{2}(n)\right]$. Let $n=1$.

Step 2. Iterate the system one time and then obtain the new value of data $=x_{2}(n+1)$ for the further calculation, where

$$
\text { Data }=\operatorname{round}\left(\operatorname{data} \times 10^{8}\right) \text {. }
$$




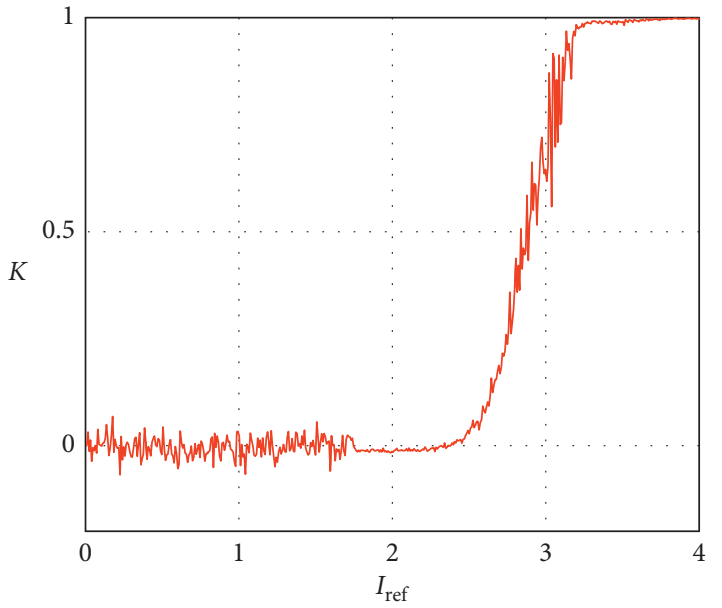

(a)

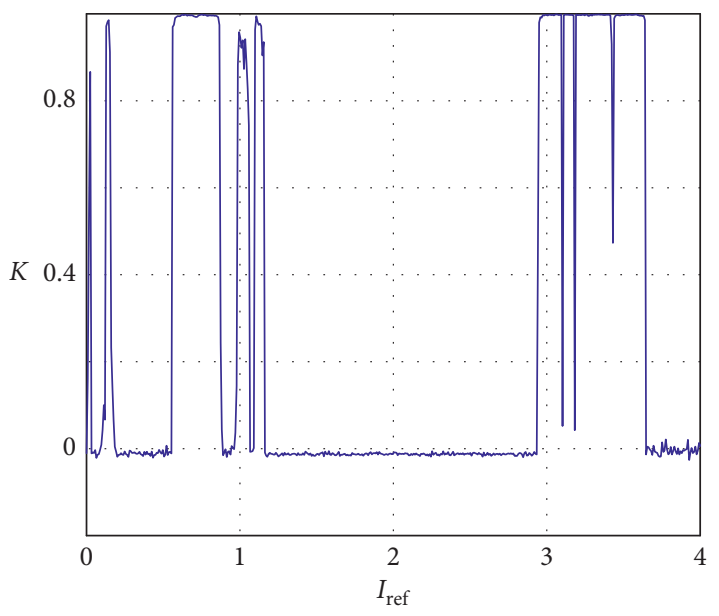

(c)

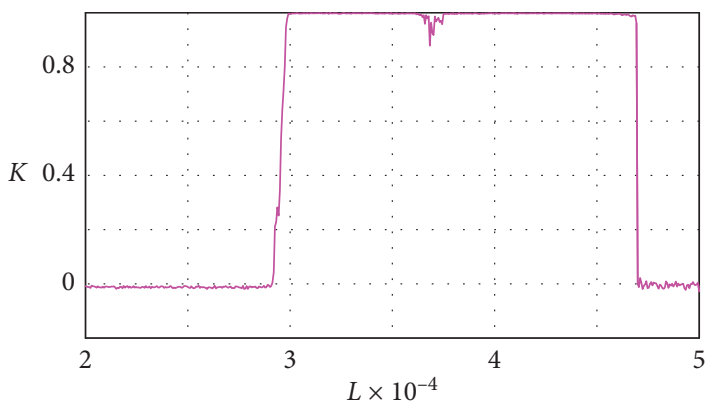

(e)

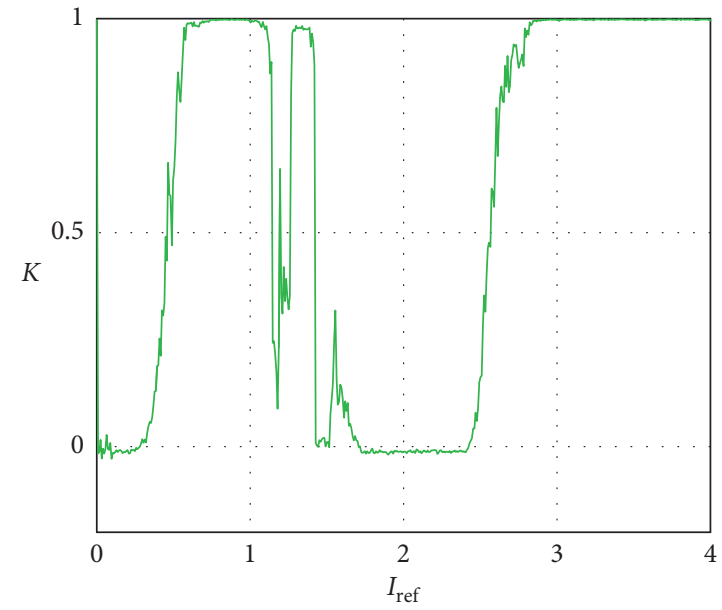

(b)

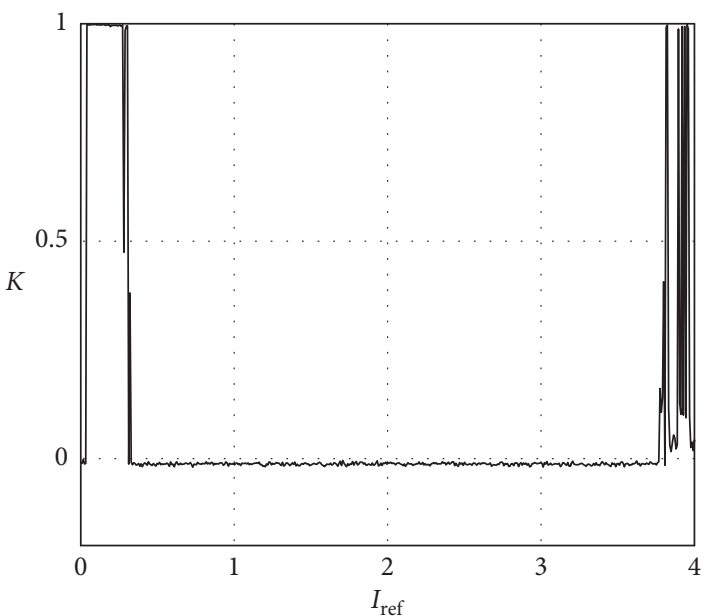

(d)

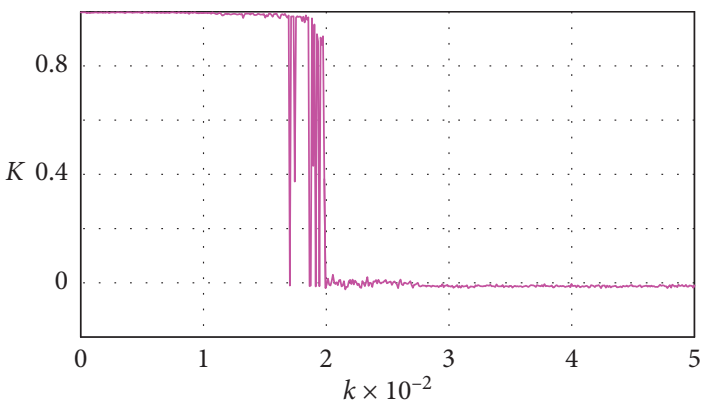

(f)

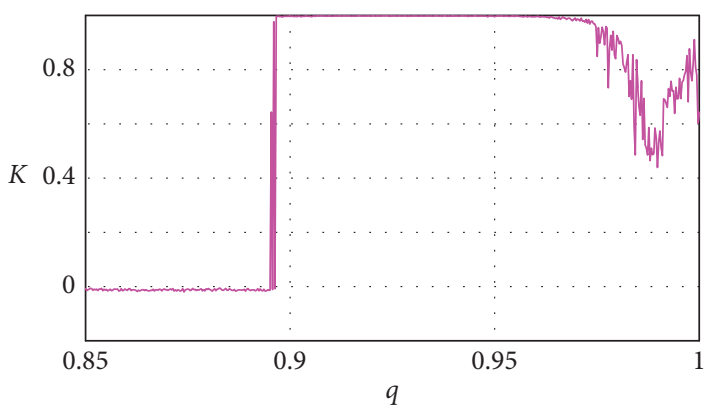

(g)

FIGURE 6: Plots of $K$ versus different parameters for the fractional-order Buck-Boost system. (a) $q=1$ and $I_{\text {ref }}$ varying, (b) $q=0.95$ and $I_{\text {ref }}$ varying, (c) $q=0.9$ and $I_{\text {ref }}$ varying, (d) $q=0.85$ and $I_{\text {ref }}$ varying, (e) $L$ varying, (f) $k$ varying, and (g) derivative order $q$ varying. 


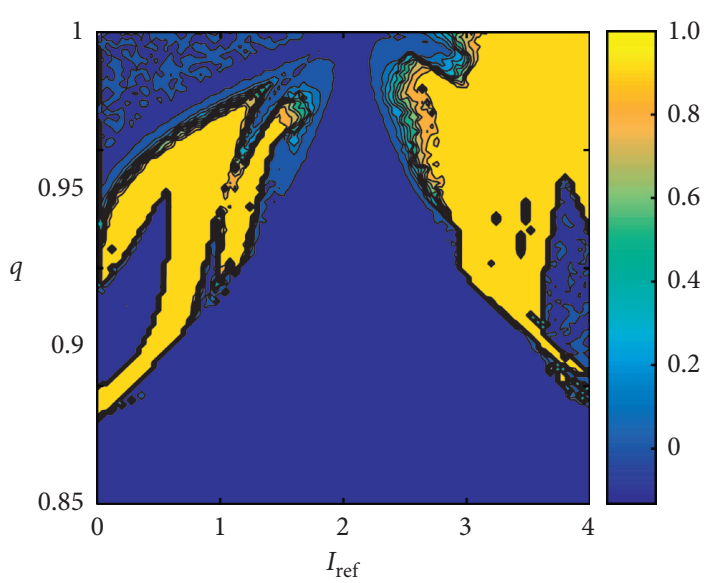

(a)

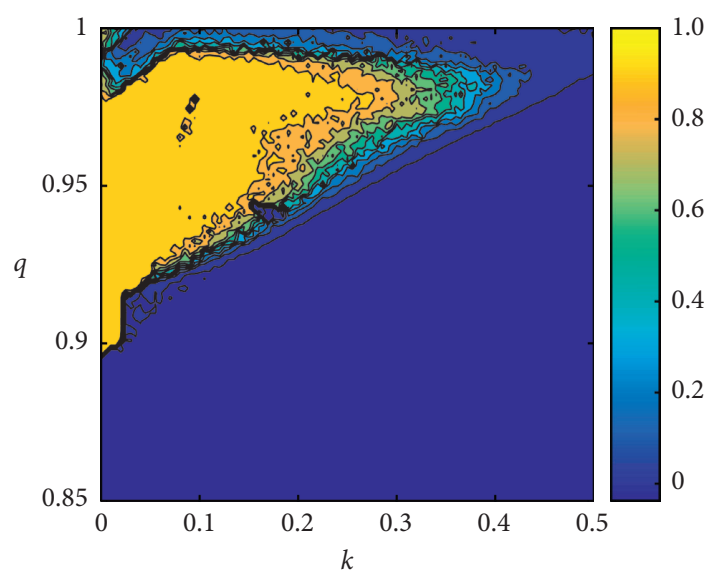

(c)

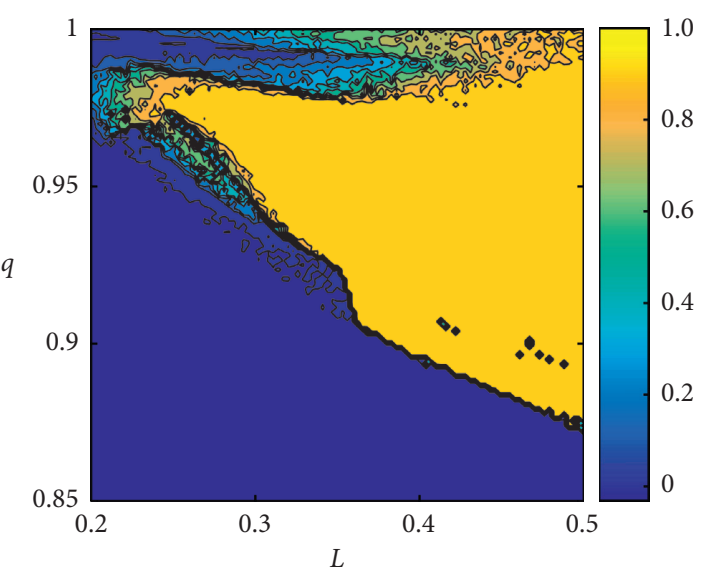

(b)

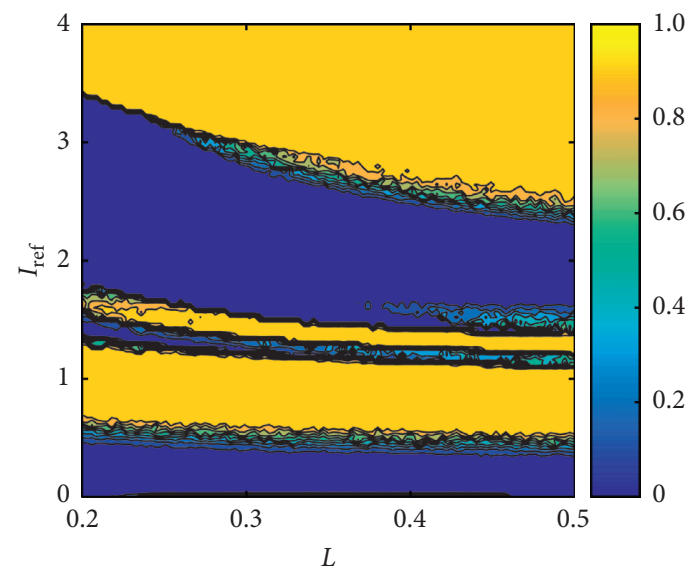

(d)

Figure 7: $K$ value-based contour plots of the of fractional-order Buck-Boost system. (a) $q-I_{\text {ref }}$, (b) $q-L$, (c) $q-k$, and (d) $I_{\text {ref }}-L$.

Thus data are converted to as a 64-bit binary number $\mathrm{DB}_{63}-\mathrm{DB}_{0}$.

Step 3. Define data $=\mathrm{DB}_{7}-\mathrm{DB}_{0}$. Then, the 8-bit 0-1 sequence data ${ }_{1}$ are saved in the target txt file.

Step 4. Set $n=n+1$. If $n \% 1000=0$, we define

$$
\left\{\begin{array}{l}
\Delta_{1}=\frac{\left[x_{1}(n) \times 10^{8}\right] \% 255}{100000}, \\
\Delta_{2}=\frac{\left[x_{2}(n) \times 10^{8}\right] \% 255}{100000}
\end{array}\right.
$$

otherwise, $\Delta_{1}=0$ and $\Delta_{2}=0$, where [.] represents the round function. Now, set the initial condition for the next iterative as

$$
\mathbf{x}_{0}=\left[x_{1}(n)+\Delta_{1}, x_{2}(n)+\Delta_{2}\right]
$$

Step 5. Do Steps 2-4 in a loop until $n>M$.

As a result, a txt file which contains $10^{8}$ more bits of "0" and " 1 " is obtained. In this section, the statistical test suite of NIST [50] is applied to test the randomness of the sequence.

The two indicators of the uniformity of $p$ values and the proportion of passing sequences are used to check whether the targeting sequence passes the standard test or not. Here, the minimum uniformity of the $p$ value is set as 0.0001 . If all $p$ values are larger than 0.0001 and the confidence interval satisfies

$$
\left[(1-\alpha)-3 \sqrt{\frac{(1-\alpha) \alpha}{m}},(1-\alpha)+3 \sqrt{\frac{(1-\alpha) \alpha}{m}}\right],
$$

where $m$ is the sample size and $\alpha$ is the given significance level, then the pseudorandom bit generator is determined to pass the standard test successfully.

One hundred pseudorandom sequences are tested, all of which are $10^{6}$ bits long. For most tests, the confidence 


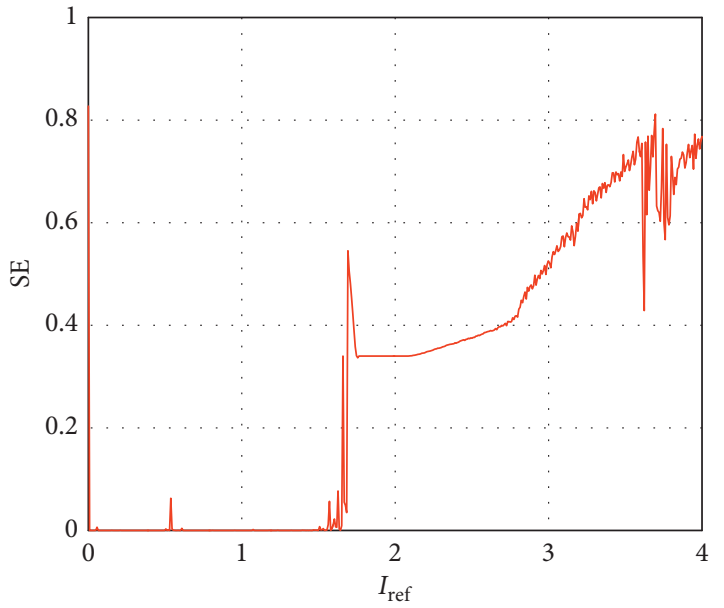

(a)

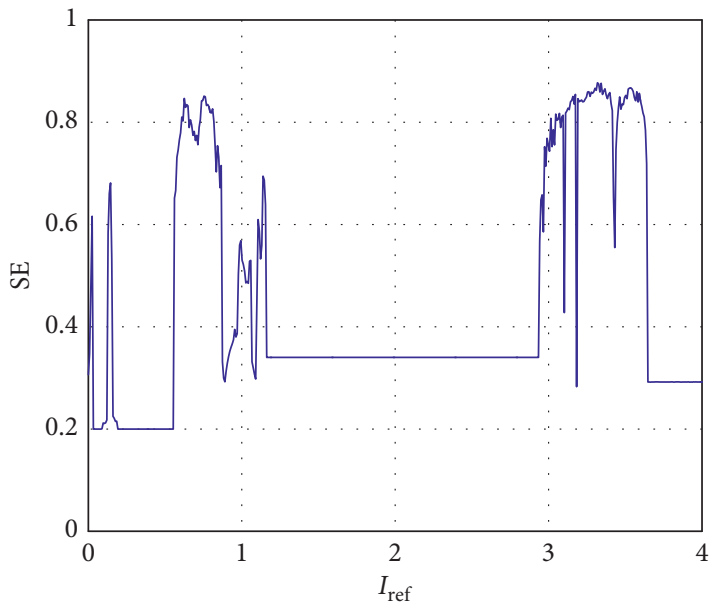

(c)

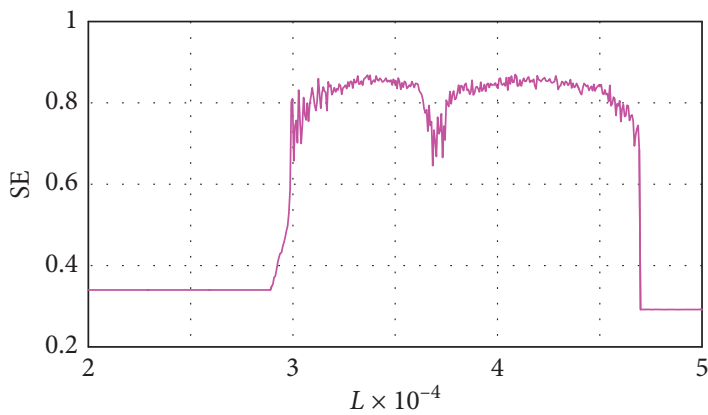

(e)

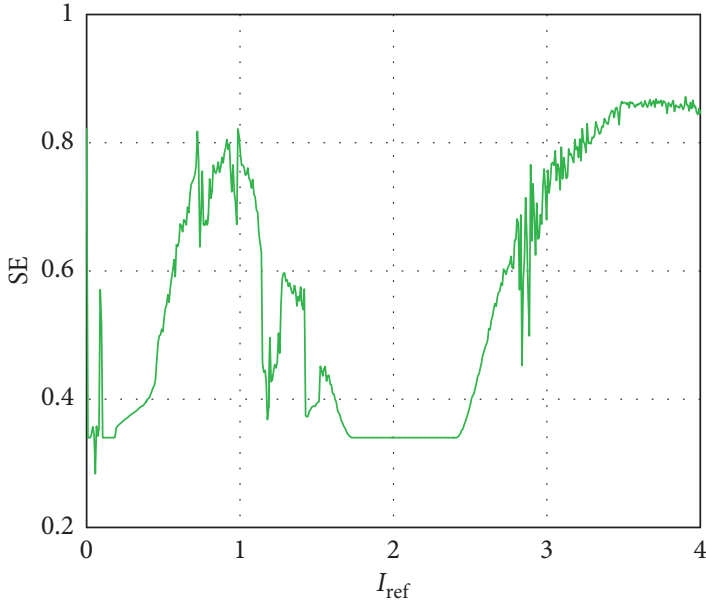

(b)

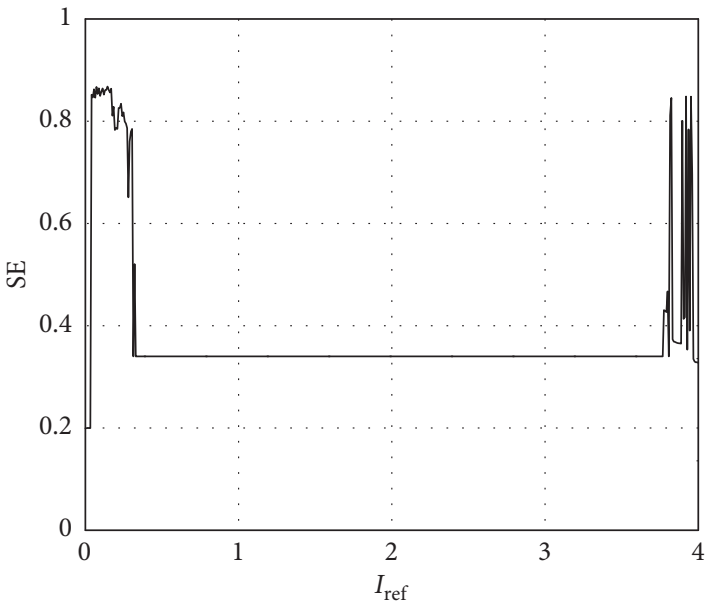

(d)

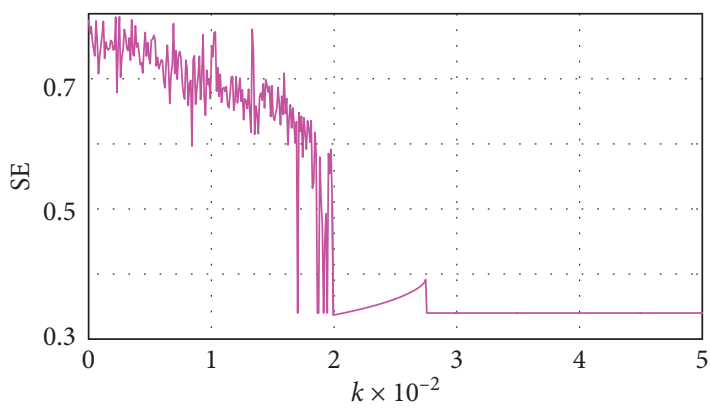

(f)

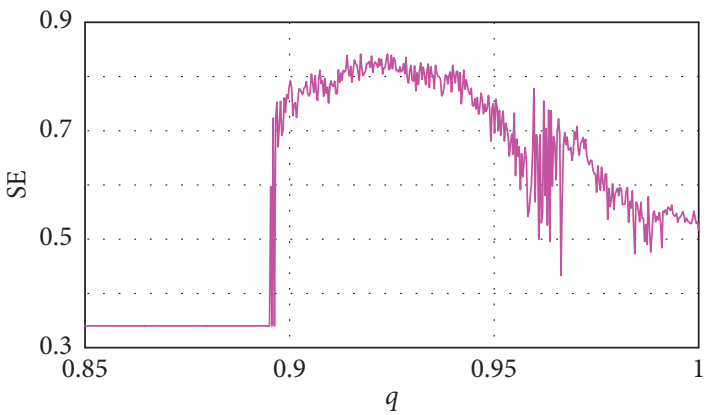

(g)

FIGURE 8: Plots of SE versus different parameters for the fractional-order Buck-Boost system. (a) $q=1$ and $I_{\text {ref }}$ varying, (b) $q=0.95$ and $I_{\text {ref }}$ varying, (c) $q=0.9$ and $I_{\text {ref }}$ varying, (d) $q=0.85$ and $I_{\text {ref }}$ varying, (e) $L$ varying, (f) $k$ varying, and (g) derivative order $q$ varying. 


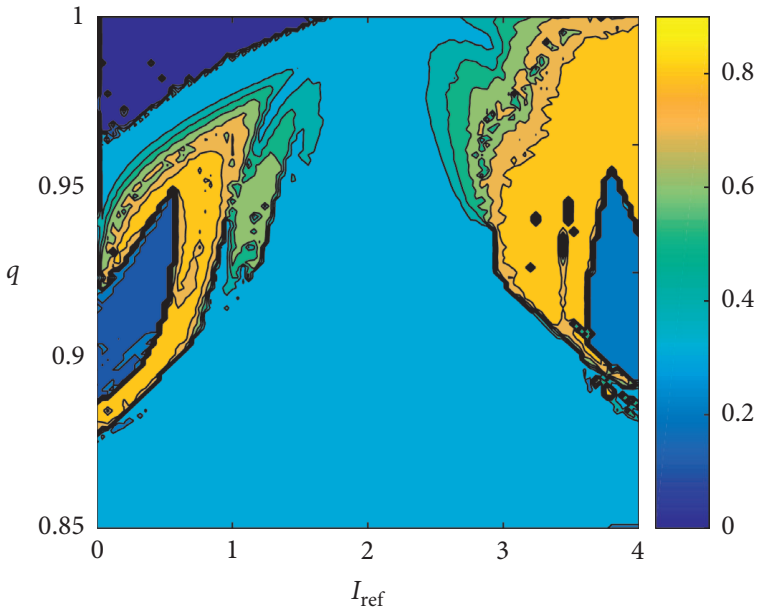

(a)

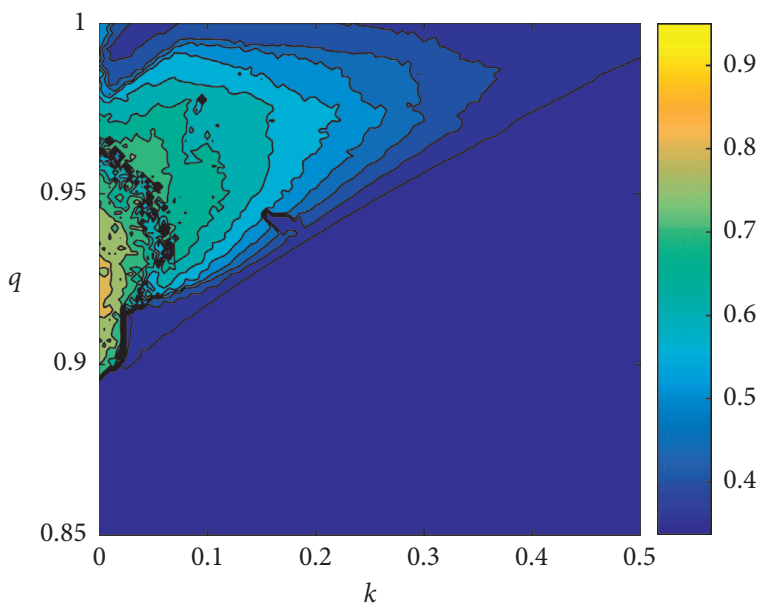

(c)

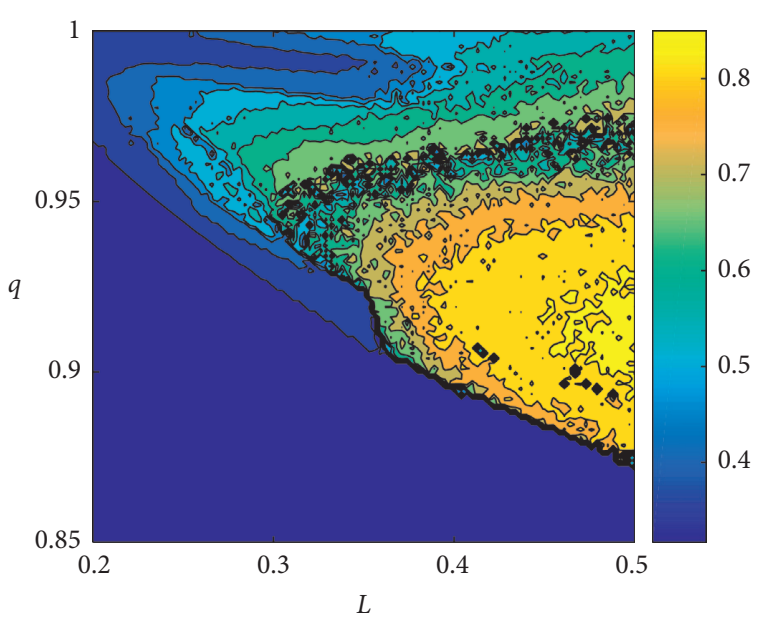

(b)

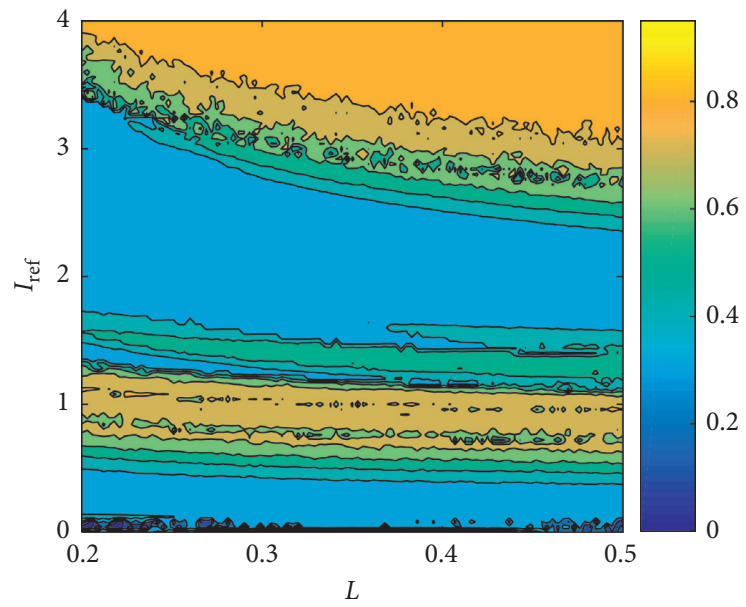

(d)

Figure 9: SE-based contour plots of the fractional-order Buck-Boost system. (a) $q-I_{\text {ref }}$, (b) $q-L$, (c) $q-k$, and (d) $I_{\text {ref }}-L$.

interval is $[96.015 \%, 1]$ when $\alpha=0.01$. For those items tested more than once (for example, N. O. Temp. is tested 148 times), we only chosen the worst results. The results are shown in Table 2 . It can be seen that all $p$ values are larger than 0.0001 , and the computed proportion for each test lies inside the confidence interval. Obviously, the obtained chaotic pseudorandom sequence is random and can be used in the information security fields. And the fractional-order Buck-Boost system provides a good model for engineering applications.

\section{Control of the Buck-Boost System}

In the real applications, sometimes, it is deleterious when there appears chaos; then, we need to control the chaos [51]. However, in many cases, we need to use the chaos [36, 37]. In this section, the control of chaos in the fractional-order Buck-Boost system is discussed.

4.1. Control by the Intensity of Feedback $k$. In fact, there is no need to design an extra controller for the Buck-Boost circuit as shown in Figure 1. As shown in the above analysis results, in real applications, the values of different parameters should be chosen carefully. According to Figures 7 and 9, if parameters are chosen in those blue regions, the system is nonchaotic, and the behaviors of the circuit is under control. Moreover, if the parameters of circuit are given but it is chaotic, the system can be controlled by increasing the value of the intensity of feedback $k$. Here, a numerical simulation is carried out on the control of chaos of the circuit by changing the value of $k$. When $U_{\text {in }}=8 \mathrm{~V}, R=10 \Omega$, $L=0.3 \mathrm{mH}, C=40 \mu \mathrm{F}, T=50 \mu \mathrm{s}, I_{\text {ref }}=3 \mathrm{~A}$, and $q=0.9$, the system is chaotic for $k=0$. In this case, we change the value of $k$ as 0.1 (for the case $q=0.9$ ) and 0.4 (for the case $q=1$ ) when $t=0.005 \mathrm{~s}$. As shown in Figure 10, the system is chaotic at the beginning, but it becomes periodic when the value of $k$ is changed.

As shown above, the control parameter can change the state of the system from chaos to periodic state. But it depends on how does the parameter affects dynamics of the system. As for the other parameters, they can be also used to control the state of the fractional-order Buck-Boost system. It should be noted out that the parameter control cannot 
TABLE 2: NIST test result of the generated pseudorandom sequence.

\begin{tabular}{lccc}
\hline & $p$ value & Proportion (\%) & \\
\hline Frequency & 0.779188 & 98 & Success \\
B. Frequency & 0.514124 & 100 & $\sqrt{ }$ \\
C. Sums (2) & 0.319084 & 98 & $\sqrt{ }$ \\
Runs & 0.419021 & 98 & $\sqrt{ }$ \\
Longest run & 0.051942 & 97 & $\sqrt{ }$ \\
Rank & 0.366918 & 99 & $\sqrt{ }$ \\
FFT & 0.102526 & 100 & $\sqrt{ }$ \\
N. O. Temp. (148) & 0.003447 & 96 & $\sqrt{ }$ \\
O. Temp. & 0.534146 & 99 & $\sqrt{ }$ \\
Universal & 0.657933 & 100 & $\sqrt{ }$ \\
App. Entropy & 0.162606 & 99 & $\sqrt{ }$ \\
R. Excur. (8) & 0.327854 & 98.6 & $\sqrt{ }$ \\
R. Excur. V. (18) & 0.017912 & 97.3 & $\sqrt{ }$ \\
Serial (2) & 0.437274 & 99 & $\sqrt{ }$ \\
L. Complexity & 0.574903 & 100 & $\sqrt{ }$ \\
\hline
\end{tabular}

make the system stable or make the system to a target state. Thus, we still need another reliable control method.

4.2. Hard Limiter Control. Hard limiter control is originally designed to control the dynamics of one-dimensional discrete maps. Specifically, Gueron [52] designed this controller to reformatting the dynamical behaviors of the discrete dynamical systems effectively. Based on the limiter control method, a one-dimensional chaotic map $x_{n+1}=f\left(x_{n}\right)$ becomes

$$
f\left(x_{n}, \rho\right)=\min \left\{f\left(x_{n}\right), \rho\right\},
$$

where $\rho$ is the value of the limiter. Recently, He et al. [53] introduced this method to control the dynamics of a fractional-order SIR epidemic model which is a two- dimensional model. For the Buck-Boost circuit, we can introduce a current limiter as shown in Figure 11 to control the dynamics of the whole circuit.

In Figure 11, the current limiter is actually a hard limiter controller. As a result, this is also a new application of the hard limiter controller. The new circuit with a hard limiter controller is rebuilt as follows.

First, let the model of Case 1 be given by

$$
\left[\begin{array}{c}
\tilde{x}_{1}\left(t_{n+1}, T\right) \\
\tilde{x}_{2}\left(t_{n+1}, T\right)
\end{array}\right]=f_{1}\left(\mathbf{x}\left(t_{n}\right), T, q\right) .
$$

In this case, the result is obtained based on the sample length of $T$.

Second, the model of Case 2 is defined as

$$
\left[\begin{array}{c}
\hat{x}_{1}\left(t_{n+1}\right) \\
\widehat{x}_{2}\left(t_{n+1}\right)
\end{array}\right]=f_{2}\left(\left[\begin{array}{c}
\min \left(\tilde{x}_{1}\left(t_{n+1}, d\left(t_{n}\right) T\right), \rho\right) \\
\tilde{x}_{2}\left(t_{n+1}, d\left(t_{n}\right) T\right)
\end{array}\right],\left(1-d\left(t_{n}\right)\right) T, q\right),
$$

where a hard limiter is used for $t \in\left(0 t_{n}, t_{n}+d_{n} T\right]$.

Third, the hard limiter controlled model with limited variable $x_{1}$ is denoted as

$$
\mathbf{x}\left(t_{n+1}\right)=\left\{\begin{array}{cl}
{\left[\begin{array}{c}
\min \left\{\tilde{x}_{1}\left(t_{n+1}, T\right), \rho\right\} \\
\tilde{x}_{2}\left(t_{n+1}\right)
\end{array}\right],} & \text { if } x_{1}\left(t_{n}\right)<I_{b}\left(t_{n}\right), \\
{\left[\begin{array}{c}
\min \left\{\hat{x}_{2}\left(t_{n+1}\right), \rho\right\} \\
\hat{x}_{2}\left(t_{n+1}\right)
\end{array}\right],} & \text { if } x_{1}\left(t_{n}\right) \geq I_{b}\left(t_{n}\right) .
\end{array}\right.
$$

Obviously, the model is proposed based on the numerical simulation model as given by equation (15). There are two major reasons for this. One is that this numerical simulation model is a kind of equivalence of the original fractional-order Buck-Boost system. The second reason is that the hard limiter controller is proposed based on the discrete map.
Let $U_{\text {in }}=8 \mathrm{~V}, R=10 \Omega, L=0.3 \mathrm{mH}, C=40 \mu \mathrm{F}, T=50 \mu \mathrm{s}$, $I_{\text {ref }}=3 \mathrm{~A}, k=0$, and $q=0.9$, and the system is chaotic. Hard limiter control results of the fractional-order Buck-Boost system with $\rho$ varying are shown in Figure 12. Bifurcation diagrams and the mean densities of the variables $x_{1}$ and $x_{2}$ show that the system has different states with the increase of the value of the limiter $\rho$. Here, the mean densities are the mean values of the variables. When the bifurcation diagram and its mean densities are overlapped, the system is convergent. Otherwise, the system is chaotic or periodic. It is shown in Figure 12 that the controlled system is convergent to the limiter $\rho$ when $\rho<2.4$. Meanwhile, it is verified in Figures 13(a) and 13(b) that the system is stable when $\rho=1$ and $\rho=2$. As for the variable $x_{1}$, it stabilizes at $x_{1}=1$ and 2, while the variable $x_{2}$ is convergent to zero. As shown in Figure 12, the system is periodic or chaotic when $\rho>2.4$. It is also shown in Figures 13(c) and 13(d) that the controlled system is periodic for $\rho=2.5$ and is chaotic for $\rho=4.5$. 


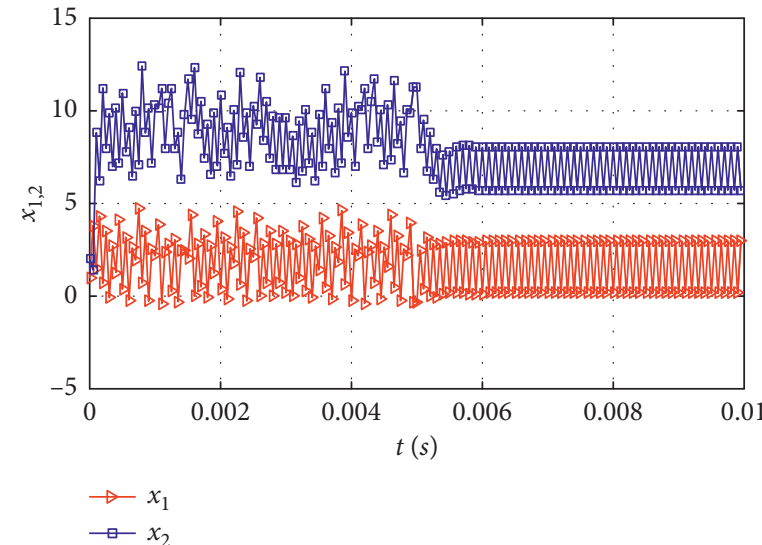

(a)

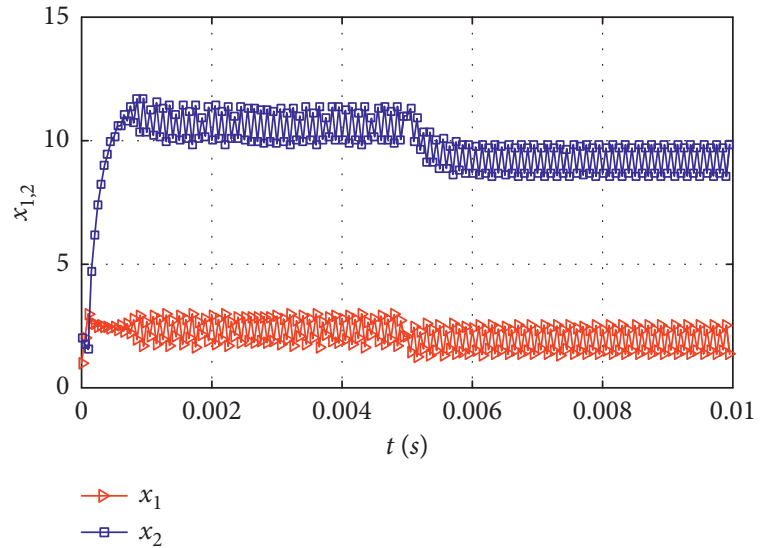

(b)

Figure 10: Plots of the fractional-order Buck-Boost system with regulable $k$. (a) $q=0.9$ and $k$ becomes to 0.1 ; (b) $q=1$ and $k$ becomes to 0.4 .

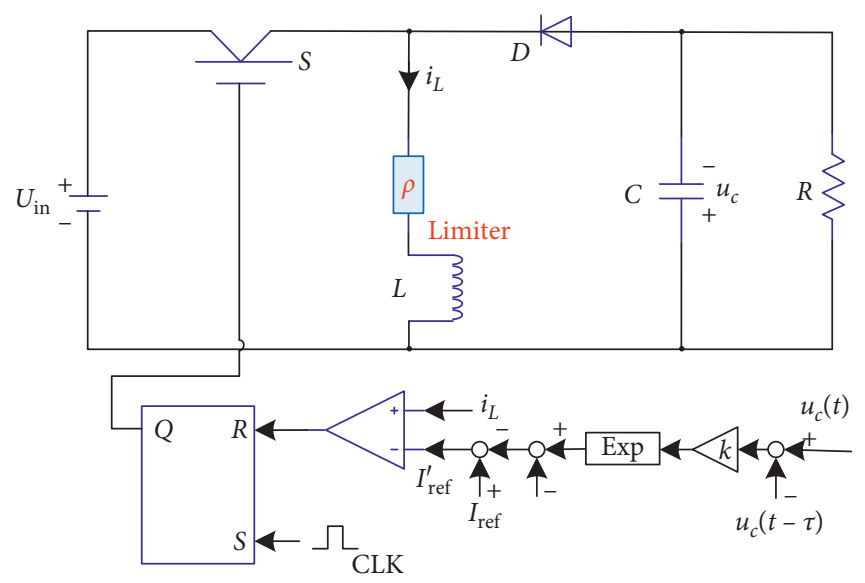

FIgURE 11: The Buck-Boost circuit with a limiter controller.

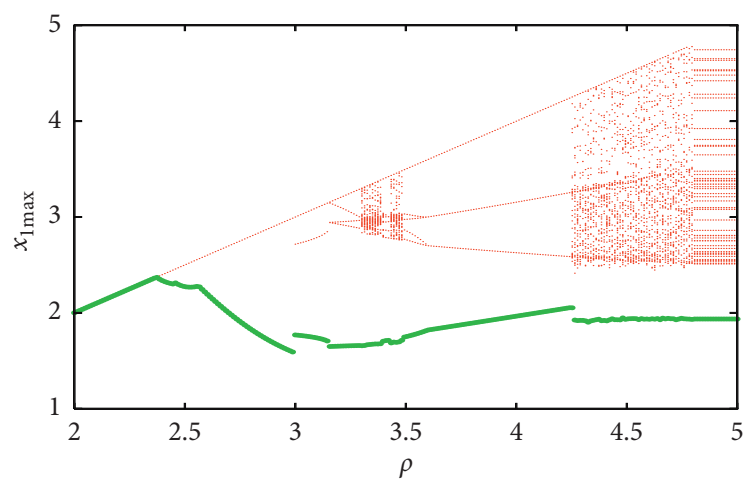

(a)

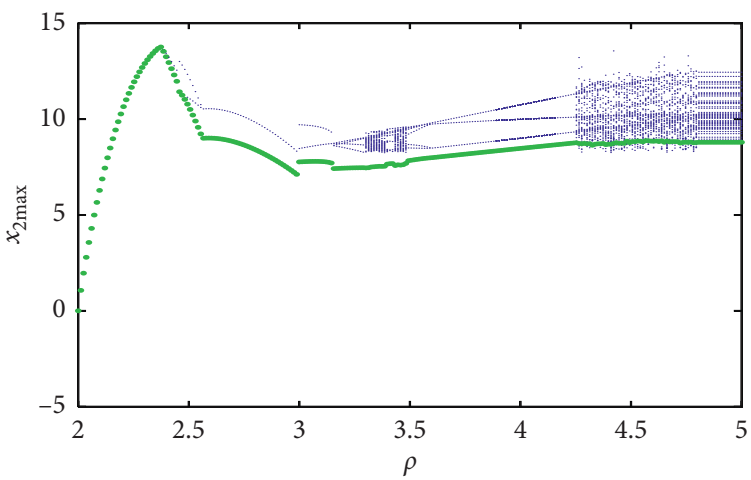

(b)

FiguRE 12: Hard limiter control results of the fractional-order Buck-Boost system with $\rho$ varying. (a) Bifurcation diagram and mean densities of $x_{1}$. (b) Bifurcation diagram and mean densities with $x_{2}$. 

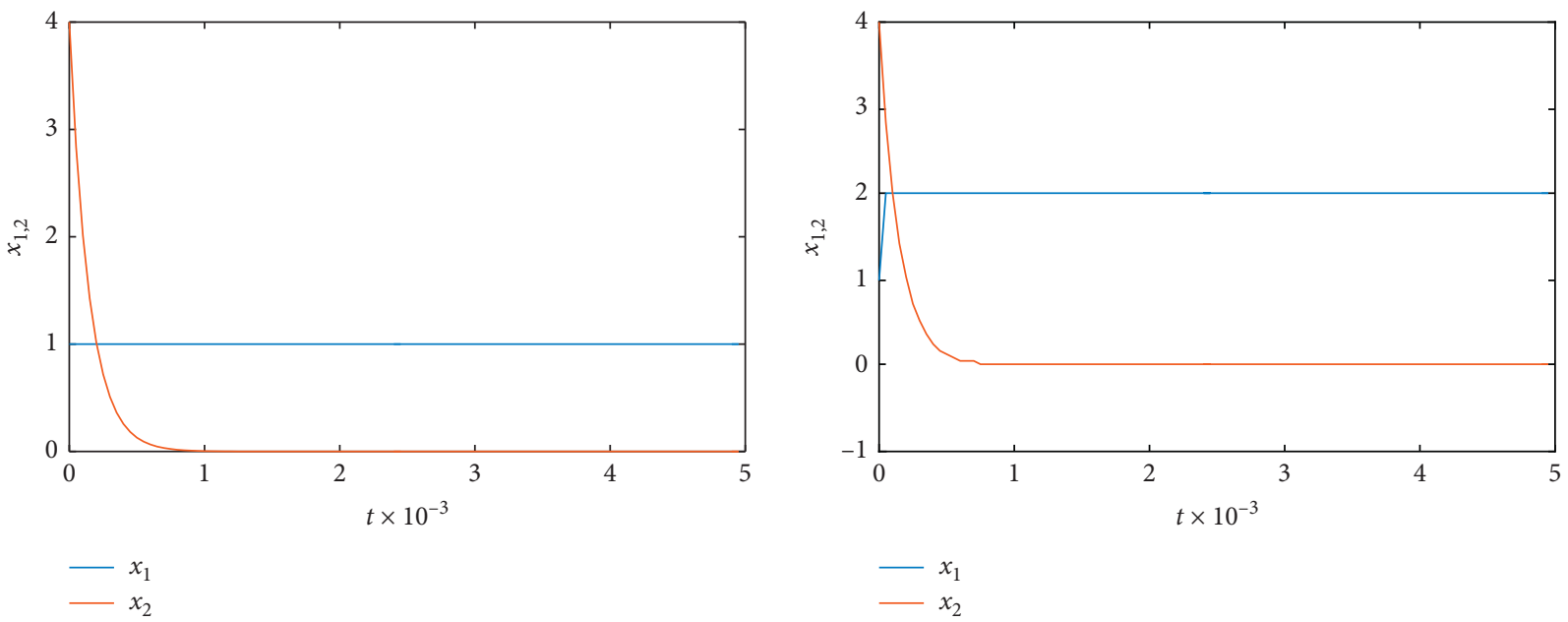

(a)

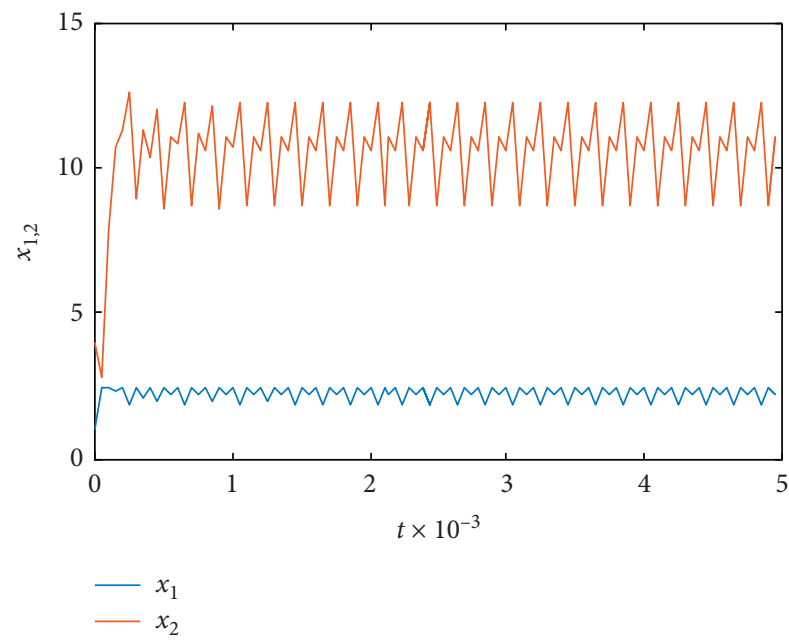

(c)

(b)

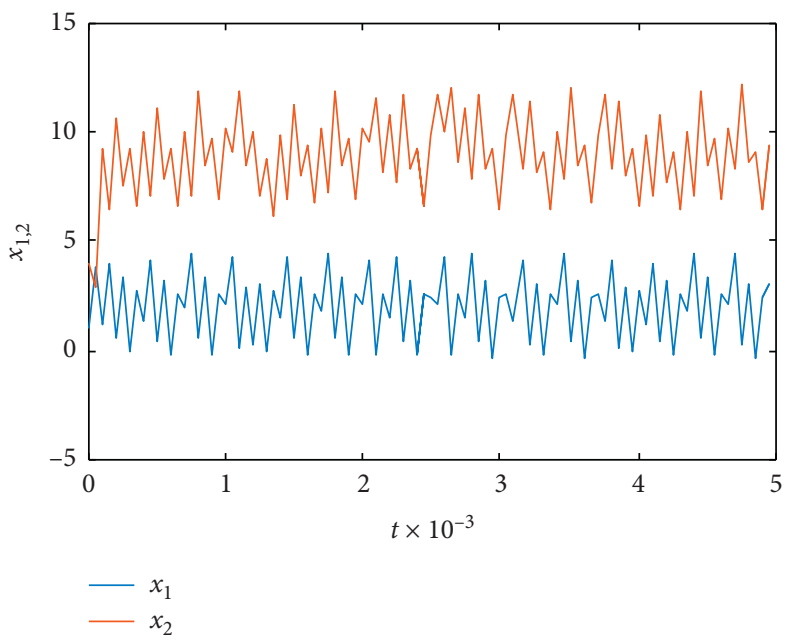

(d)

FiguRE 13: Hard limiter control results of the fractional-order Buck-Boost system with different $\rho$. (a) $\rho=1$, (b) $\rho=2$, (c) $\rho=2.5$, and (d) $\rho=4.5$.

According to above analysis, it is found that the hard limiter controller is effective for controlling the fractionalorder Buck-Boost system. First, it is easy to realize in the real applications since we only need to add a current limiter in the circuit. Second, compared with the control by the intensity of feedback $k$, the hard limiter control is more flexible. The system can be controlled to convergent state, periodic state, and chaotic state by properly choosing a limiter $\rho$. Third, the hard limit controller is designed based on the obtained numerical model. In our opinion, the numerical solution is obtained from the system equation; thus, it also provides a model for analysis of the fractional-order Buck-Boost system. As for the hard limiter controller, it is designed for the discrete system, originally. Thus, it is reasonable to introduce the hard limiter controller to the numerical model of the system. Meanwhile, the proposed model can well explain the proposed circuit as shown in
Figure 11. In conclusion, the hard limiter control can be related to the real situations and has a strong application prospect for controlling the Buck-Boost circuit.

\section{Conclusions}

In this study, a fractional-order Buck-Boost system is deduced and analyzed based on the Adomian decomposition method. Chaos, complexity, and application of this fractional-order Buck-Boost system are investigated, and methods including bifurcation diagram, the $0-1$ test, and SE are introduced to carry out the study. According to the simulations and analyses, the following conclusions are obtained.

(1) The deduced fractional order has rich dynamics with different parameters, which means that the original 
Buck-Boost converter could be chaotic if a set of proper parameters is given.

(2) As shown in those contour plots, the system has wide regions for chaos. The $0-1$ test is an effective tool for detecting chaos, but SE shows more information on the variation tendency of the system with the variations of different parameters. Meanwhile, it shows that the generated pseudorandom sequence is random since it passes all the NIST tests.

(3) Chaos of the circuit can be controlled by adjusting the intensity of feedback $k$ or by introducing a hard limiter controller.

Thus, this study extended the model of the Buck-Boost converter by introducing the fractional calculus. And it provides a good model for engineering applications in the information security field. Our next work is to investigate the hard limiter control of the circuit practically and to use this system for the information encryption.

\section{Data Availability}

The data used to support the findings of this study are included within the article.

\section{Conflicts of Interest}

The authors declare that there are no conflicts of interest.

\section{Acknowledgments}

This work was supported by the Hunan Provincial Department of Education General Project Fund (No. 18C0743), the Natural Science Foundation of China (Nos. 61901530, 62071496, and 62061008), and the Natural Science Foundation of Hunan Province (No. 2020JJ5767).

\section{References}

[1] E. Maali and B. Vahidi, "Double-deck buck-boost converter with soft switching operation," IEEE Transactions on Power Electronics, vol. 31, no. 6, pp. 4324-4330, 2016.

[2] S. Singh, N. Rathore, and D. Fulwani, "Mitigation of negative impedance instabilities in a DC/DC buck-boost converter with composite load," Journal of Power Electronics, vol. 16, no. 3, pp. 1046-1055, 2016.

[3] M. Salimi and S. Siami, "Cascade nonlinear control of dc-dc buck/boost converter using exact feedback linearization," in Proceedings of the 2015 4th International Conference on Electric Power and Energy Conversion Systems (EPECS), pp. 1-5, IEEE, Sharjah, UAE, November 2015.

[4] M. K. Kavitha and A. Kavitha, "Nonlinear analysis of hysteretic modulation-based sliding mode controlled quadratic buck-boost converter," Journal of Circuits, Systems and Computers, vol. 28, no. 02, Article ID 1950025, 2019.

[5] A. El Aroudi, L. Benadero, E. Toribio, and S. Machiche, "Quasiperiodicity and chaos in the dc-dc buck-boost converter," International Journal of Bifurcation and Chaos, vol. 10 , no. 02 , pp. $359-371,2000$.

[6] K. W. E. Cheng, M. Liu, and J. Wu, "Chaos study and parameter-space analysis of the DC-DC buck-boost converter,"
IEEE Proceedings-Electric Power Applications, vol. 150, no. 2, pp. 126-138, 2003.

[7] M. R. Banaei and H. A. F. Bonab, "A high efficiency nonisolated buck-boost converter based on zeta converter," IEEE Transactions on Industrial Electronics, vol. 67, pp. 1991-1998, 2020.

[8] A. El Aroudi, L. Benadero, E. Toribio, and G. Olivar, "Hopf bifurcation and chaos from torus breakdown in a pwm voltage-controlled dc-dc boost converter," IEEE Transactions on Circuits and Systems I: Fundamental Theory and Applications, vol. 46, no. 11, pp. 1374-1382, 1999.

[9] J. D. Morcillo, D. Burbano, and F. Angulo, "Adaptive ramp technique for controlling chaos and subharmonic oscillations in DC-DC power converters," IEEE Transactions on Power Electronics, vol. 31, no. 7, pp. 5330-5343, 2016.

[10] Y. Wang, R. Yang, B. Zhang, and W. Hu, "Smale horseshoes and symbolic dynamics in the buck-boost DC-DC converter," IEEE Transactions on Industrial Electronics, vol. 65, no. 1, pp. 800-809, 2018.

[11] Ş. Demirbaş, H. Fidanboy, and E. Kurt, "Exploration of the chaotic behaviour in a buck-boost converter depending on the converter and load elements," Journal of Electronic Materials, vol. 45, pp. 3889-3899, 2016.

[12] M. P. Aghababa, "Stabilization of a class of cascade nonlinear switched systems with application to chaotic systems," International Journal of Robust and Nonlinear Control, vol. 28, no. 11, pp. 3640-3656, 2018.

[13] F. Yalcin, U. Arifoglu, I. Yazici, and K. Erin, "Robust singlephase inverter based on the buck-boost converter through an efficient hybrid control," Iet Power Electronics, vol. 13, no. 1, pp. 50-59, 2020.

[14] A. Kavitha and G. Uma, "Resonant parametric perturbation method to control chaos in current mode controlled dc-dc buck-boost converter," Journal of Electrical Engineering and Technology, vol. 5, no. 1, pp. 171-178, 2010.

[15] P. Sriramalakshmi, A. Kavitha, P. Sanjeevikumar, T. Sutikno, P. Kiran Maroti, and V. K. Ramachandaramurthy, "Control of chaos in a current mode controlled buck boost converter using weak periodic perturbation method," International Journal of Power Electronics and Drive Systems (IJPEDS), vol. 8, no. 4, pp. 1467-1480, 2017.

[16] J. R. Sharma and D. Kumar, "A fast and efficient composite Newton-Chebyshev method for systems of nonlinear equations," Journal of Complexity, vol. 49, pp. 56-73, 2018.

[17] N. Giménez and G. Matera, "On the bit complexity of polynomial system solving," Journal of Complexity, vol. 51, pp. 20-67, 2019.

[18] S. G. Samko, A. A. Kilbas, and O. I. Marichev, Fractional Integrals and Derivatives: Theory and Applications, CRC, Boca Raton, FL, USA, 1993.

[19] H. Rudolf, Applications of Fractional Calculus in Physics, World Scientific, Singapore, 2000.

[20] H. Sun, Y. Zhang, D. Baleanu, W. Chen, and Y. Chen, "A new collection of real world applications of fractional calculus in science and engineering," Communications in Nonlinear Science and Numerical Simulation, vol. 64, pp. 213-231, 2018.

[21] B. Yan, S. He, and S. Wang, "Multistability in a fractionalorder centrifugal flywheel governor system and its adaptive control," Complexity, vol. 2020, Article ID 8844657, 2020.

[22] S. Kumar, R. Kumar, C. Cattani, and B. Samet, "Chaotic behaviour of fractional predator-prey dynamical system," Chaos, Solitons \& Fractals, vol. 135, Article ID 109811, 2020. 
[23] S. He, K. Sun, and Y. Peng, "Detecting chaos in fractionalorder nonlinear systems using the smaller alignment index," Physics Letters A, vol. 383, no. 19, pp. 2267-2271, 2019.

[24] S. Bendoukha and S. Abdelmalek, "The fractional chua chaotic system: dynamics, synchronization, and application to secure communications," International Journal of Nonlinear Sciences and Numerical Simulation, vol. 20, no. 1, pp. 77-88, 2019.

[25] Q. Zhidong, T. Juntao, P. Jin et al., "Fractional controller design of a dc-dc converter for pemfc," IEEE Access, vol. 8, pp. 120134-120144, 2020.

[26] A. G. Radwan, A. A. Emira, A. M. AbdelAty, and A. T. Azar, "Modeling and analysis of fractional order dc-dc converter," ISA Transactions, vol. 82, pp. 184-199, 2018.

[27] D. Vanitha and M. Rathinakumar, "Fractional order pid controlled pv buck boost converter with coupled inductor," International Journal of Power Electronics and Drive Systems, vol. 8, p. 1409, 2017.

[28] Y. Ningning, W. Chaojun, J. Rong, and L. Chongxin, "Modeling and characteristics analysis for a buck-boost converter in pseudo-continuous conduction mode based on fractional calculus," Mathematical Problems in Engineering, vol. 2016, Article ID 6835910, , 2016.

[29] Q. Lai, Z. Wan, L. K. Kengne, P. D. K. Kuate, and C. Chen, "Two-memristor-based chaotic system with infinite coexisting attractors," IEEE Transactions on Circuits and Systems II: Express Briefs, p. 1, 2020.

[30] Q. Lai, Z. Wan, P. D. Kamdem Kuate, and H. Fotsin, "Coexisting attractors, circuit implementation and synchronization control of a new chaotic system evolved from the simplest memristor chaotic circuit," Communications in Nonlinear Science and Numerical Simulation, vol. 89, Article ID 105341, 2020.

[31] Q. Lai, B. Norouzi, and F. Liu, "Dynamic analysis, circuit realization, control design and image encryption application of an extended Lü system with coexisting attractors," Chaos, Solitons \& Fractals, vol. 114, pp. 230-245, 2018.

[32] Q. Lai, "A unified chaotic system with various coexisting attractors," International Journal of Bifurcation and Chaos, vol. 31, no. 01, Article ID 2150013, 2021.

[33] S. He, K. Sun, and H. Wang, "Complexity analysis and dsp implementation of the fractional-order lorenz hyperchaotic system," Entropy, vol. 17, no. 12, pp. 8299-8311, 2015.

[34] Z. Wu and X. P. Zhang, "Chaos control of buck-boost converter in current mode," Proceedings of the CSU-EPSA, vol. 30, no. 8, pp. 51-57, 2018.

[35] X. Yi, R. Guo, and Y. Qi, "Stabilization of chaotic systems with both uncertainty and disturbance by the ude-based control method," IEEE Access, vol. 8, pp. 62471-62477, 2020.

[36] L. M. Zhang, K. H. Sun, W. H. Liu, and S. B. He, "A novel color image encryption scheme using fractional-order hyperchaotic system and dna sequence operations," Chinese Physics B, vol. 26, Article ID 100504, 2017.

[37] F. F. Yang, J. Mou, C. F. Luo, and Y. H. Cao, "An improved color image encryption scheme and cryptanalysis based on hyperchaotic sequence," Physica Scripta, vol. 94, Article ID 085206, 2019.

[38] B. Dumitru, D. Kai, and S. Enrico, Fractional Calculus: Models and Numerical Methods, World Scientific, Singapore, 2012.

[39] G. Adomian and R. Rach, "Modified adomian polynomials," Mathematical and Computer Modelling, vol. 24, no. 11, pp. 39-46, 1996.

[40] R. Caponetto and S. Fazzino, "An application of adomian decomposition for analysis of fractional-order chaotic systems," International Journal of Bifurcation and Chaos, vol. 23, no. 03, Article ID 1350050, 2013.

[41] D. Cafagna and G. Grassi, "Bifurcation and chaos in the fractional-order chen system via a time-domain approach," International Journal of Bifurcation and Chaos, vol. 18, no. 07, pp. 1845-1863, 2008.

[42] I. Falconer, G. A. Gottwald, I. Melbourne, and K. Wormnes, "Application of the 0-1 test for chaos to experimental data," SIAM Journal on Applied Dynamical Systems, vol. 6, no. 2, pp. 395-402, 2007.

[43] G. A. Gottwald and I. Melbourne, "On the implementation of the 0-1 test for chaos," SIAM Journal on Applied Dynamical Systems, vol. 8, no. 1, pp. 129-145, 2009.

[44] Z. Wang, A. Akgul, V.-T. Pham, and S. Jafari, "Chaos-based application of a novel no-equilibrium chaotic system with coexisting attractors," Nonlinear Dynamics, vol. 89, no. 3, pp. 1877-1887, 2017.

[45] Z. Aram, S. Jafari, J. Ma, J. C. Sprott, S. Zendehrouh, and V.-T. Pham, "Using chaotic artificial neural networks to model memory in the brain," Communications in Nonlinear Science and Numerical Simulation, vol. 44, pp. 449-459, 2017.

[46] S. T. Kingni, V.-T. Pham, S. Jafari, and P. Woafo, "A chaotic system with an infinite number of equilibrium points located on a line and on a hyperbola and its fractional-order form," Chaos, Solitons \& Fractals, vol. 99, pp. 209-218, 2017.

[47] R. Hamza, "A novel pseudo random sequence generator for image-cryptographic applications," Journal of Information Security and Applications, vol. 35, pp. 119-127, 2017.

[48] H. Xu, X. Tong, and X. Meng, "An efficient chaos pseudorandom number generator applied to video encryption," Optik, vol. 127, no. 20, pp. 9305-9319, 2016.

[49] A. A. Rezk, A. H. Madian, A. G. Radwan, and A. M. Soliman, "Reconfigurable chaotic pseudo random number generator based on fpga," AEU-International Journal of Electronics and Communications, vol. 98, pp. 174-180, 2019.

[50] A. Rukhin, J. Soto, and J. Nechvata, "A Statistical Test Suite for Random and Pseudorandom Number Generators for Cryptographic Applications," Technical Report, DTIC Document, 2001.

[51] G. Chen and X. Yu, Chaos Control: Theory and Applications, Springer Science, Berlin, Germany, 2003.

[52] S. Gueron, "Controlling one-dimensional unimodal population maps by harvesting at a constant rate," Physical Review E, vol. 57, no. 3, p. 3645, 1998.

[53] S. He and S. Banerjee, "Epidemic outbreaks and its control using a fractional order model with seasonality and stochastic infection," Physica A: Statistical Mechanics and Its Applications, vol. 501, pp. 408-417, 2018. 